\title{
Explicit Determinantal Representation Formulas of $W$-Weighted Drazin Inverse Solutions of Some Matrix Equations over the Quaternion Skew Field
}

\author{
Ivan I. Kyrchei \\ Pidstrygach Institute for Applied Problems of Mechanics and Mathematics, NAS of Ukraine, Lviv 79060, Ukraine \\ Correspondence should be addressed to Ivan I. Kyrchei; kyrchei@online.ua
}

Received 25 January 2016; Accepted 24 April 2016

Academic Editor: Romain Aubry

Copyright (C) 2016 Ivan I. Kyrchei. This is an open access article distributed under the Creative Commons Attribution License, which permits unrestricted use, distribution, and reproduction in any medium, provided the original work is properly cited.

By using determinantal representations of the $W$-weighted Drazin inverse previously obtained by the author within the framework of the theory of the column-row determinants, we get explicit formulas for determinantal representations of the $W$-weighted Drazin inverse solutions (analogs of Cramer's rule) of the quaternion matrix equations WAWX $=\mathbf{D}, \mathbf{X W B W}=\mathbf{D}$, and $\mathbf{W}_{1} \mathrm{AW}_{1} \mathrm{XW}_{2} \mathbf{B W}_{2}=\mathbf{D}$.

\section{Introduction}

Throughout the paper, we denote the real number field by $\mathbb{R}$, the set of all $m \times n$ matrices over the quaternion algebra

$$
\begin{aligned}
\mathbb{W} & =\left\{a_{0}+a_{1} i+a_{2} j+a_{3} k \mid i^{2}=j^{2}=k^{2}\right. \\
& \left.=-1, a_{0}, a_{1}, a_{2}, a_{3} \in \mathbb{R}\right\}
\end{aligned}
$$

by $\mathbb{U}^{m \times n}$, and the set of all $m \times n$ matrices over $\mathbb{W}$ with a rank $r$ by $\mathbb{U}_{r}^{m \times n}$. Let $M(n, \mathbb{U})$ be the ring of $n \times n$ quaternion matrices. For $\mathbf{A} \in \mathbb{Q}^{n \times m}$, the symbol $\mathbf{A}^{*}$ stands for the conjugate transpose (Hermitian adjoint) matrix of $\mathbf{A}$. The matrix $\mathbf{A}=$ $\left(a_{i j}\right) \in \mathbb{Q}^{n \times n}$ is Hermitian if $\mathbf{A}^{*}=\mathbf{A}$.

For $\mathbf{A} \in \mathbb{Q}^{m \times n}$, we denote by

$\mathscr{R}_{r}(\mathbf{A})=\left\{\mathbf{y} \in \mathbb{U}^{m}: \mathbf{y}=\mathbf{A x}, \mathbf{x} \in \mathbb{U}^{n}\right\}$ the column right space of $\mathbf{A}$;

$\mathcal{N}_{r}(\mathbf{A})=\left\{\mathbf{y} \in \mathbb{\mathbb { T } ^ { n }}: \mathbf{A} \mathbf{x}=0\right\}$ the right null space of $\mathbf{A}$;

$\mathscr{R}_{l}(\mathbf{A})=\left\{\mathbf{y} \in \mathbb{Q}^{n}: \mathbf{y}=\mathbf{x A}, \mathbf{x} \in \mathbb{T}^{m}\right\}$ the column left space of $\mathbf{A}$;

$\mathcal{N}_{r}(\mathbf{A})=\left\{\mathbf{y} \in \mathbb{\boxplus}^{m}: \mathbf{x A}=0\right\}$ the left null space of $\mathbf{A}$.

In the past, researches into the quaternion skew field had a more theoretical importance, but now a growing number of investigations give wide practical applications of quaternions. In particular through their attitude orientation, the quaternions arise in various fields such as quaternionic quantum theory [1], fluid mechanics and particle dynamics $[2,3]$, computer graphics [4], aircraft orientation [5], robotic systems [6], and life science [7, 8].

Research on quaternion matrix equations and generalized inverses, which are usefulness tools used to solve matrix equations, has been actively ongoing for more recent years. We mention only some recent papers. Yuan et al. [9] derived solutions of the quaternion matrix equation $A X=B$ and their applications in color image restoration. Wang et al. [10] studied extreme ranks of real matrices in solution of the quaternion matrix equation $A X B=C$. Yuan et al. [11] obtained the expressions of least squares Hermitian solution with minimum norm of the quaternion matrix equation $(A X B, C X D)=(E, F)$. Feng and Cheng [12] gave a clear description of the solution set to the quaternion matrix equation $A X-\bar{X} B=0$. Jiang and Wei [13] derived the explicit solution of the quaternion matrix equation $X-A \widetilde{X} B=C$. Caiqin et al. [14] obtained the expressions of the explicit solutions of quaternion matrix equations $X F-A X=B Y$ and $X F-A \widetilde{X}=B Y$. Yuan and Wang [15] gave the expressions of the least squares $\eta$-Hermitian solution with the least norm of the quaternion matrix equation $A X B+C X D=E$. Zhang 
et al. derived [16] the expressions of the minimal norm least squares solution, the pure imaginary least squares solution, and the real least squares solution for the quaternion matrix equation $A X=B$.

The definitions of the generalized inverse matrices have been extended to quaternion matrices as follows.

The Moore-Penrose inverse of $\mathbf{A} \in \mathbb{H}^{m \times n}$, denoted by $\mathbf{A}^{\dagger}$, is the unique matrix $\mathbf{X} \in \mathbb{H}^{n \times m}$ satisfying the following equations:

$$
\begin{aligned}
\mathbf{A X A} & =\mathbf{A} \\
\mathbf{X A X} & =\mathbf{X} \\
(\mathbf{A X})^{*} & =\mathbf{A X} \\
(\mathbf{X A})^{*} & =\mathbf{X A}
\end{aligned}
$$

For $\mathbf{A} \in \mathbb{H}^{n \times n}$ with $k=$ Ind $\mathbf{A}$ being the smallest positive number such that rank $\mathbf{A}^{k+1}=\operatorname{rank} \mathbf{A}^{k}$, the Drazin inverse of $\mathbf{A}$, denoted by $\mathbf{A}^{D}$, is defined to be the unique matrix $\mathbf{X}$ that satisfies (3) and the equations

$$
\begin{aligned}
\mathbf{A X} & =\mathbf{X} \mathbf{A} ; \\
\mathbf{A}^{k+1} \mathbf{X} & =\mathbf{A}^{k} .
\end{aligned}
$$

In particular, when Ind $\mathbf{A}=1$, then $\mathbf{X}$ is called the group inverse of $\mathbf{A}$ and is denoted by $\mathbf{X}=\mathbf{A}^{g}$. If Ind $\mathbf{A}=0$, then $\mathbf{A}$ is nonsingular, and $\mathbf{A}^{D} \equiv \mathbf{A}^{\dagger}=\mathbf{A}^{-1}$.

Cline and Greville [17] extended the Drazin inverse of square matrix to rectangular matrix that has been generalized to the quaternion algebra as follows.

For $\mathbf{A} \in \mathbb{H}^{m \times n}$ and $\mathbf{W} \in \mathbb{H}^{n \times m}$, the $W$-weighted Drazin inverse of $\mathbf{A}$ with respect to $\mathbf{W}$ is the unique solution to the following equations:

$$
\begin{aligned}
(\mathbf{A W})^{k+1} \mathbf{X W} & =(\mathbf{A W})^{k} ; \\
\mathbf{X W A W X} & =\mathbf{X} ; \\
\mathbf{A W X} & =\mathbf{X W A},
\end{aligned}
$$

where $k=\max \{\operatorname{Ind}(\mathbf{A W}), \operatorname{Ind}(\mathbf{W A})\}$.

The Drazin inverse and weighted Drazin inverse have several important applications such as applications in singular differential and difference equations [18], signal processing [19], Markov chains and statistic problems [20, 21], descriptor continuous-time systems [22], numerical analysis and Kronecker product systems [23], solving singular fuzzy linear system [24, 25], constrained linear systems [26], and so forth.

Cramer's rule for the $W$-weighted Drazin inverse solutions, in particular, has been derived in [27] for singular linear equations and in [26] for a class of restricted matrix equations. Recently, within the framework of the theory of the column-row determinants, Song [28] has first obtained a determinantal representation of the $W$-weighted Drazin inverse and Cramer's rule of a class of restricted matrix equations over the quaternion algebra. But in obtaining, he has used auxiliary matrices other than that are given. In [29], we have obtained new determinantal representations of the
$W$-weighted Drazin inverse over the quaternion skew field without any auxiliary matrices.

An important application of determinantal representations of generalized inverses is the Cramer rule for generalized inverse solutions of matrix equations.

But when is there a need for a $W$-weighted Drazin inverse solution? Consider, for example, the following matrix equation: $\mathbf{A}_{1} \mathbf{X}=\mathbf{D}$. Let $\mathbf{A}_{1}$ be rectangular and we can represent it as $\mathbf{A}_{1}=\mathbf{W A W}$, where $\mathbf{W A}$ and $\mathbf{A W}$ are quadratic and singular. Furthermore, we have the following restrictions: $\mathscr{R}_{r}(\mathbf{X}) \subset \mathscr{R}_{r}\left((\mathbf{A W})^{k}\right), \mathscr{N}_{l}(\mathbf{X}) \supset \mathscr{N}_{l}\left((\mathbf{W A})^{k}\right)$. Then its $W$ weighted Drazin inverse solution is needed.

In the paper we investigate analogs of Cramer's rule for $W$-weighted Drazin inverse solutions of the following quaternion matrix equations:

$$
\begin{array}{r}
\text { WAWX }=\mathbf{D}, \\
\mathbf{X W B W}^{=} \mathbf{D}, \\
\mathbf{W}_{1} \mathrm{AW}_{1} \mathbf{X W}_{2} \mathbf{B W}_{2}=\mathrm{D} .
\end{array}
$$

The paper is organized as follows. We start with introducing of the row-column determinants and determinantal representations of the Moore-Penrose and Drazin inverses for a quaternion matrix obtained by them in Section 2.1. Determinantal representations of the $W$-weighted Drazin inverse and its properties were considered in Section 2.2. In Section 3.1, we give the background of the problem of Cramer's rule for the $W$-weighted Drazin inverse solution. In Section 3.2 we obtain explicit representation formulas of the $W$-weighted Drazin inverse solutions (analogs of Cramer's rule) of the quaternion matrix equation (10). Consequently, we get both similar and special determinantal representation formulas of the $W$-weighted Drazin inverse solutions of (8) and (9). In Section 4, we give numerical examples to illustrate the main result.

\section{Preliminaries}

2.1. Determinantal Representations of the Moore-Penrose and Drazin Inverses by the Column and Row Determinants. The theory of the row-column determinants over the quaternion skew field has been introduced in [30-32], and later it has been applied to research generalized inverses and generalized inverse solutions of matrix equations. In particular, determinantal representations of the Moore-Penrose [33, 34] and explicit representation formulas for the minimum norm least squares solutions of some quaternion matrix equations [35] and determinantal representations of the Drazin [36] and $W$-weighted Drazin inverses [29] have been obtained by the author. Song derived determinantal representation of the generalized inverse $A_{T, S}^{2}$ [37], the Bott-Duffin inverse [38], the Cramer rule for the solutions of restricted matrix equations [39], the generalized Stein quaternion matrix equation [40], and so forth.

For $\mathbf{A}=\left(a_{i j}\right) \in M(n, \mathbb{U})$ we define $n$ row determinants and $n$ column determinants as follows.

Suppose that $S_{n}$ is the symmetric group on the set $I_{n}=$ $\{1, \ldots, n\}$. 
Definition 1. The $i$ th row determinant of $\mathbf{A}=\left(a_{i j}\right) \in M(n, \mathbb{H})$ is defined for all $i=\overline{1, n}$ by putting

$$
\begin{aligned}
& \operatorname{rdet}_{i} \mathbf{A}=\sum_{\sigma \in S_{n}}(-1)^{n-r} \\
& \cdot a_{i i_{k_{1}}} a_{i_{k_{1}} i_{k_{1}+1}} \cdots a_{i_{k_{1}+l_{1}}} \cdots a_{i_{k_{r}} i_{k_{r}+1}} \cdots a_{i_{k_{r}+l_{r}} i_{k_{r}}}, \\
& \sigma=\left(i i_{k_{1}} i_{k_{1}+1} \cdots i_{k_{1}+l_{1}}\right) \\
& \cdot\left(i_{k_{2}} i_{k_{2}+1} \cdots i_{k_{2}+l_{2}}\right) \cdots\left(i_{k_{r}} i_{k_{r}+1} \cdots i_{k_{r}+l_{r}}\right),
\end{aligned}
$$

with conditions $i_{k_{2}}<i_{k_{3}}<\cdots<i_{k_{r}}$ and $i_{k_{t}}<i_{k_{t}+s}$ for $t=\overline{2, r}$ and $s=\overline{1, l_{t}}$.

Definition 2. The $j$ th column determinant of $\mathbf{A}=\left(a_{i j}\right) \in$ $M(n, \mathbb{H})$ is defined for all $j=\overline{1, n}$ by putting

$$
\begin{aligned}
& \operatorname{cdet}_{j} \mathbf{A}=\sum_{\tau \in S_{n}}(-1)^{n-r} \\
& \quad \cdot a_{j_{k_{r}} j_{k_{r}+l_{r}}} \cdots a_{j_{k_{r}+1} i_{k_{r}}} \cdots a_{j j_{k_{1}+l_{1}}} \cdots a_{j_{k_{1}+1} j_{k_{1}}} a_{j_{k_{1}} j}, \\
& \tau=\left(j_{k_{r}+l_{r}} \cdots j_{k_{r}+1} j_{k_{r}}\right) \cdots\left(j_{k_{2}+l_{2}} \cdots j_{k_{2}+1} j_{k_{2}}\right) \\
& \quad \cdot\left(j_{k_{1}+l_{1}} \cdots j_{k_{1}+1} j_{k_{1}} j\right),
\end{aligned}
$$

with conditions $j_{k_{2}}<j_{k_{3}}<\cdots<j_{k_{r}}$ and $j_{k_{t}}<j_{k_{t}+s}$ for $t=\overline{2, r}$ and $s=\overline{1, l_{t}}$.

Suppose that $\mathbf{A}^{i j}$ denotes the submatrix of $\mathbf{A}$ obtained by deleting both the $i$ th row and the $j$ th column. Let $\mathbf{a}_{. j}$ be the $j$ th column and let $\mathbf{a}_{i}$. be the $i$ th row of $\mathbf{A}$. Suppose that $\mathbf{A}_{\cdot j}$ (b) denotes the matrix obtained from $\mathbf{A}$ by replacing its $j$ th column with the column $\mathbf{b}$ and that $\mathbf{A}_{i}$. (b) denotes the matrix obtained from $\mathbf{A}$ by replacing its $i$ th row with the row $\mathbf{b}$.

The following theorem has a key value in the theory of the column and row determinants.

Theorem 3 (see [30]). If $\mathbf{A}=\left(a_{i j}\right) \in M(n, \mathbb{H})$ is Hermitian, then $\operatorname{rdet}_{1} \mathbf{A}=\cdots=\operatorname{rdet}_{n} \mathbf{A}=\operatorname{cdet}_{1} \mathbf{A}=\cdots=\operatorname{cdet}_{n} \mathbf{A} \in \mathbb{R}$.

Since all column and row determinants of a Hermitian matrix over $\mathbb{W}$ are equal, we can define the determinant of a Hermitian matrix $\mathbf{A} \in M(n, \mathbb{H})$. By definition, we put $\operatorname{det} \mathbf{A}:=\operatorname{rdet}_{i} \mathbf{A}=\operatorname{cdet}_{i} \mathbf{A}$, for all $i=\overline{1, n}$. The determinant of a Hermitian matrix has properties similar to a usual determinant. They are completely explored in $[30,31]$ by its row and column determinants. In particular, within the framework of the theory of the column-row determinants, we have the determinantal representation of the inverse matrix over $\mathbb{W}$ by analogs of classical adjoint matrix. Further, we consider determinantal representations of generalized inverses obtained by the column-row determinants.

We will use the following notations. Let $\alpha$ := $\left\{\alpha_{1}, \ldots, \alpha_{k}\right\} \subseteq\{1, \ldots, m\}$ and $\beta:=\left\{\beta_{1}, \ldots, \beta_{k}\right\} \subseteq\{1, \ldots, n\}$ be subsets of the order $1 \leq k \leq \min \{m, n\}$. By $\mathbf{A}_{\beta}^{\alpha}$ denote the submatrix of $\mathbf{A}$ determined by the rows indexed by $\alpha$ and the columns indexed by $\beta$. Then $\mathbf{A}_{\alpha}^{\alpha}$ denotes the principal submatrix determined by the rows and columns indexed by $\alpha$. If $\mathbf{A} \in M(n, \mathbb{H})$ is Hermitian, then by $\left|\mathbf{A}_{\alpha}^{\alpha}\right|$ denote the corresponding principal minor of $\operatorname{det} \mathbf{A}$. For $1 \leq k \leq n$, the collection of strictly increasing sequences of $k$ integers chosen from $\{1, \ldots, n\}$ is denoted by $L_{k, n}:=\left\{\alpha: \alpha=\left(\alpha_{1}, \ldots, \alpha_{k}\right), 1 \leq \alpha_{1} \leq \cdots \leq \alpha_{k} \leq n\right\}$. For fixed $i \in \alpha$ and $j \in \beta$, let $I_{r, m}\{i\}:=\left\{\alpha: \alpha \in L_{r, m}, i \in \alpha\right\}, J_{r, n}\{j\}:=$ $\left\{\beta: \beta \in L_{r, n}, j \in \beta\right\}$.

Denote by $\mathbf{a}_{. j}^{*}$ and $\mathbf{a}_{i}^{*}$. the $j$ th column and the $i$ th row of $\mathbf{A}^{*}$ and by $\mathbf{a}_{\cdot j}^{(m)}$ and $\mathbf{a}_{i .}^{(m)}$ the $j$ th column and the $i$ th row of $\mathbf{A}^{m}$, respectively.

The following theorem gives determinantal representations of the Moore-Penrose inverse over the quaternion skew field $\mathbb{H}$.

Theorem 4 (see [32]). If $\mathbf{A} \in \mathbb{M}_{r}^{m \times n}$, then the MoorePenrose inverse $\mathbf{A}^{+}=\left(a_{i j}^{+}\right) \in \mathbb{U}^{n \times m}$ possesses the following determinantal representations:

$$
a_{i j}^{+}=\frac{\sum_{\beta \in J_{r, n}\{i\}} \operatorname{cdet}_{i}\left(\left(\mathbf{A}^{*} \mathbf{A}\right)_{\cdot i}\left(\mathbf{a}_{\cdot j}^{*}\right)\right)_{\beta}^{\beta}}{\sum_{\beta \in J_{r, n}}\left|\left(\mathbf{A}^{*} \mathbf{A}\right)_{\beta}^{\beta}\right|}
$$

or

$$
a_{i j}^{+}=\frac{\sum_{\alpha \in I_{r, m}\{j\}} \operatorname{rdet}_{j}\left(\left(\mathbf{A A}^{*}\right)_{j .}\left(\mathbf{a}_{i \cdot}^{*}\right)\right)_{\alpha}^{\alpha}}{\sum_{\alpha \in I_{r, m}}\left|\left(\mathbf{A} \mathbf{A}^{*}\right)_{\alpha}^{\alpha}\right|}
$$

for all $i=\overline{1, n}$ and $j=\overline{1, m}$

Proposition 5 (see [20]). If $\operatorname{Ind}(\mathbf{A})=k$, then $\mathbf{A}^{D}=$ $\mathbf{A}^{k}\left(\mathbf{A}^{2 k+1}\right)^{+} \mathbf{A}^{k}$.

Denote by $\widehat{\mathbf{a}}_{\cdot s}$ and $\check{\mathbf{a}}_{t}$ the $s$ th column of $\left(\mathbf{A}^{2 k+1}\right)^{*} \mathbf{A}^{k}=$ : $\widehat{\mathbf{A}}=\left(\widehat{a}_{i j}\right) \in \mathbb{H}^{n \times n}$ and the $t$ th row of $\mathbf{A}^{k}\left(\mathbf{A}^{2 k+1}\right)^{*}=: \check{\mathbf{A}}=$ $\left(\check{a}_{i j}\right) \in \mathbb{H}^{n \times n}$, respectively, for all $s, t=\overline{1, n}$. Using the determinantal representations of the Moore-Penrose inverse (13) and (14) and Proposition 5, the following determinantal representations of the Drazin inverse for an arbitrary square matrix over $\mathbb{H}$ have been obtained in [33].

Theorem 6 (see [33]). If $\mathbf{A} \in M(n, \mathbb{H})$ with Ind $\mathbf{A}=k$ and $\operatorname{rank} \mathbf{A}^{k+1}=\operatorname{rank} \mathbf{A}^{k}=r$, then the Drazin inverse $\mathbf{A}^{D}$ possesses the following determinantal representations:

$$
\begin{aligned}
& a_{i j}^{D} \\
& =\frac{\sum_{t=1}^{n} a_{i t}^{(k)} \sum_{\beta \in J_{r, n}\{t\}} \operatorname{cdet}_{t}\left(\left(\mathbf{A}^{2 k+1}\right)^{*}\left(\mathbf{A}^{2 k+1}\right)_{\cdot t}\left(\widehat{\mathbf{a}}_{\cdot j}\right)\right)_{\beta}^{\beta}}{\sum_{\beta \in J_{r, n}}\left|\left(\mathbf{A}^{2 k+1}\right)^{*}\left(\mathbf{A}^{2 k+1}\right)_{\beta}^{\beta}\right|}, \\
& a_{i j}^{D} \\
& =\frac{\sum_{s=1}^{n}\left(\sum_{\alpha \in I_{r, n}\{s\}} \operatorname{rdet}_{s}\left(\left(\mathbf{A}^{2 k+1}\left(\mathbf{A}^{2 k+1}\right)^{*}\right)_{\cdot s}\left(\check{\mathbf{a}}_{i \cdot}\right)\right)_{\alpha}^{\alpha}\right) a_{s j}^{(k)}}{\sum_{\alpha \in I_{r, n}}\left|\left(\mathbf{A}^{2 k+1}\left(\mathbf{A}^{2 k+1}\right)^{*}\right)_{\alpha}^{\alpha}\right|} .
\end{aligned}
$$

In the special case, when $\mathbf{A} \in M(n, \mathbb{U})$ is Hermitian, we can obtain simpler determinantal representations of the Drazin inverse. 
Theorem 7 (see [33]). If $\mathbf{A} \in M(n, \mathbb{H})$ is Hermitian with Ind $\mathbf{A}=k$ and $\operatorname{rank} \mathbf{A}^{k+1}=\operatorname{rank} \mathbf{A}^{k}=r$, then the Drazin inverse $\mathbf{A}^{D}=\left(a_{i j}^{D}\right) \in \mathbb{H}^{n \times n}$ possesses the following determinantal representations:

$$
a_{i j}^{D}=\frac{\sum_{\beta \in J_{r, n}\{i\}} \operatorname{cdet}_{i}\left(\left(\mathbf{A}^{k+1}\right)_{\cdot i}\left(\mathbf{a}_{\cdot j}^{k}\right)\right)_{\beta}^{\beta}}{\sum_{\beta \in J_{r, n}}\left|\left(\mathbf{A}^{k+1}\right)_{\beta}^{\beta}\right|}
$$

or

$$
a_{i j}^{D}=\frac{\sum_{\alpha \in I_{r, n}\{j\}} \operatorname{rdet}_{j}\left(\left(\mathbf{A}^{k+1}\right)_{j .}\left(\mathbf{a}_{i \cdot}^{(k)}\right)\right)_{\alpha}^{\alpha}}{\sum_{\alpha \in I_{r, n}}\left|\left(\mathbf{A}^{k+1}\right)_{\alpha}^{\alpha}\right|} .
$$

2.2. Determinantal Representations of the W-Weighted Drazin Inverse. We introduce some mathematical background from the theory of the $W$-weighted Drazin inverse $[27,41,42]$ that can be generalized to $\mathbb{H}$.

Lemma 8. Let $\mathbf{A} \in \mathbb{T}^{m \times n}$ and $\mathbf{W} \in \mathbb{T}^{n \times m}$ with $k=$ $\max \{\operatorname{Ind}(\mathbf{A W}), \operatorname{Ind}(\mathbf{W A})\}$. Then one has
(a) $\mathbf{A}_{d, \mathbf{W}}=\mathbf{A}\left((\mathbf{W A})^{D}\right)^{2}=\left((\mathbf{A W})^{D}\right)^{2} \mathbf{A}$;
(b) $\mathbf{A}_{d, \mathbf{W}} \mathbf{W}=(\mathbf{A W})^{D} ; \mathbf{W A}_{d, \mathbf{W}}=(\mathbf{W A})^{D}$;
(c) $\mathbf{A}_{d, \mathbf{W}}=\left\{(\mathbf{A W})^{k}\left[(\mathbf{A W})^{2 k+1}\right]^{+}(\mathbf{A W})^{k}\right\} \mathbf{W}^{+} ; \mathbf{A}_{d, \mathbf{W}}=$ $\mathbf{W}^{+}\left\{(\mathbf{W A})^{k}\left[(\mathbf{W A})^{2 k+1}\right]^{+}(\mathbf{W A})^{k}\right\}$
(d) $\mathbf{W A W A}_{d, \mathbf{W}}=\mathbf{P}_{\mathscr{R}_{r}\left((\mathbf{W A})^{k}\right), \mathcal{N}_{r}\left((\mathbf{W A})^{k}\right)} ; \mathbf{A}_{d, \mathbf{W}} \mathbf{W A W}=$ $\mathbf{P}_{\mathscr{R}_{l}\left((\mathrm{AW})^{k}\right), \mathscr{N}_{l}\left((\mathrm{AW})^{k}\right)}$,

where $\mathbf{P}_{\mathscr{R}_{r}\left((\mathbf{W A})^{k}\right), N_{r}\left((\mathbf{W A})^{k}\right)}$ is the projector on $\mathscr{R}_{r}\left((\mathbf{W A})^{k}\right)$ along $\mathscr{N}_{r}\left((\mathbf{W A})^{k}\right)$ and $P_{\mathscr{R}_{l}\left((\mathbf{A W})^{k}\right), N_{l}\left((\mathrm{AW})^{k}\right)}$ is the projector on $\mathscr{R}_{l}\left((\mathrm{AW})^{k}\right)$ along $\mathscr{N}_{l}\left((\mathrm{AW})^{k}\right)$.

In particular, the point (a) of Lemma 8 due to Cline and Greville [17] is generalized [28] to $\mathbb{H}$. Using this proposition, we have obtained [29] the following determinantal representations of $W$-weighted Drazin inverse. $\mathbb{H}^{m \times m}$

Denote $\mathbf{W A}=: \mathbf{U}=\left(u_{i j}\right) \in \mathbb{H}^{n \times n}$ and $\mathbf{A W}=: \mathbf{V}=\left(v_{i j}\right) \epsilon$

Due to Theorem 6, we denote an entry of the Drazin inverse $\mathbf{U}^{D}$ by

$$
\begin{aligned}
& u_{i j}^{D, 1} \\
& =\frac{\sum_{t=1}^{n} u_{i t}^{(k)} \sum_{\beta \in J_{r, n}\{t\}} \operatorname{cdet}_{t}\left(\left(\mathbf{U}^{2 k+1}\right)^{*}\left(\mathbf{U}^{2 k+1}\right)_{\cdot t}\left(\widehat{\mathbf{u}}_{\cdot j}\right)\right)_{\beta}^{\beta}}{\sum_{\beta \in J_{r, n}}\left|\left(\mathbf{U}^{2 k+1}\right)^{*}\left(\mathbf{U}^{2 k+1}\right)_{\beta}^{\beta}\right|}
\end{aligned}
$$

or

$$
\begin{aligned}
& u_{i j}^{D, 2} \\
& =\frac{\sum_{s=1}^{n}\left(\sum_{\alpha \in I_{r, n}\{s\}} \operatorname{rdet}_{s}\left(\left(\mathbf{U}^{2 k+1}\left(\mathbf{U}^{2 k+1}\right)^{*}\right)_{\cdot s}\left(\check{\mathbf{u}}_{i \cdot}\right)\right)_{\alpha}^{\alpha}\right) u_{s j}^{(k)}}{\sum_{\alpha \in I_{r, n}}\left|\left(\mathbf{U}^{2 k+1}\left(\mathbf{U}^{2 k+1}\right)^{*}\right)_{\alpha}^{\alpha}\right|},
\end{aligned}
$$

where $\widehat{\mathbf{u}}_{\cdot s}$ and $\check{\mathbf{u}}_{t}$. are the $s$ th column of $\left(\mathbf{U}^{2 k+1}\right)^{*} \mathbf{U}^{k}=: \widehat{\mathbf{U}}=$ $\left(\widehat{u}_{i j}\right) \in \mathbb{H}^{n \times n}$ and the $t$ th row of $\mathbf{U}^{k}\left(\mathbf{U}^{2 k+1}\right)^{*}=: \check{\mathbf{U}}=\left(\check{u}_{i j}\right) \in$ $\mathbb{H}^{n \times n}$, respectively, for all $s, t=\overline{1, n}$, and $r=\operatorname{rank} \mathbf{U}^{k+1}=$ $\operatorname{rank} \mathbf{U}^{k}$.

Then we have the following determinantal representations of $\mathbf{A}_{d, \mathbf{W}}=\left(a_{i j}^{d, \mathbf{W}}\right) \in \mathbb{H}^{m \times n}$ :

$$
a_{i j}^{d, \mathbf{w}}=\sum_{q=1}^{n} a_{i q}\left(u_{q j}^{D}\right)^{(2)},
$$

where

$$
\left(u_{q j}^{D}\right)^{(2)}=\sum_{p=1}^{n} u_{q p}^{D, l} u_{p j}^{D, f}
$$

for all $l, f=\overline{1,2}$ and $u_{i j}^{D, 1}$ from (18) and $u_{i j}^{D, 2}$ from (19).

Similarly using $\mathbf{V}=\left(v_{i j}\right) \in \mathbb{H}^{m \times m}$,

$$
a_{i j}^{d, \mathbf{w}}=\sum_{q=1}^{m}\left(v_{i q}^{D}\right)^{(2)} a_{q j},
$$

where the first factor is one of the following four possible equations:

$$
\left(v_{i q}^{D}\right)^{(2)}=\sum_{p=1}^{m} v_{i p}^{D, l} v_{p q}^{D, f}
$$

for all $l, f=\overline{1,2}$, and an entry of the Drazin inverse $\mathbf{V}^{D}$ is denoted by

$$
\begin{aligned}
& v_{i j}^{D, 1} \\
& \sum_{t=1}^{m} v_{i t}^{(k)} \sum_{\beta \in J_{r, m}\{t\}} \operatorname{cdet}_{t}\left(\left(\mathbf{V}^{2 k+1}\right)^{*}\left(\mathbf{V}^{2 k+1}\right)_{\cdot t}\left(\widehat{\mathbf{v}}_{\cdot j}\right)\right)_{\beta}^{\beta} \\
& \sum_{\beta \in J_{r, m}}\left|\left(\mathbf{V}^{2 k+1}\right)^{*}\left(\mathbf{V}^{2 k+1}\right)_{\beta}^{\beta}\right|
\end{aligned}
$$

or

$$
\begin{aligned}
& v_{i j}^{D, 2} \\
& =\frac{\sum_{s=1}^{m}\left(\sum_{\alpha \in I_{r, m}\{s\}} \operatorname{rdet}_{s}\left(\left(\mathbf{V}^{2 k+1}\left(\mathbf{V}^{2 k+1}\right)^{*}\right)_{\cdot s}\left(\check{\mathbf{v}}_{i \cdot}\right)\right)_{\alpha}^{\alpha}\right) v_{s j}^{(k)}}{\sum_{\alpha \in I_{r, m}}\left|\left(\mathbf{V}^{2 k+1}\left(\mathbf{V}^{2 k+1}\right)^{*}\right)_{\alpha}^{\alpha}\right|},
\end{aligned}
$$

where $\widehat{\mathbf{v}}_{\text {.s }}$ and $\check{\mathbf{v}}_{t}$. are the $s$ th column of $\left(\mathbf{V}^{2 k+1}\right)^{*} \mathbf{V}^{k}=: \widehat{\mathbf{V}}=$ $\left(\widehat{v}_{i j}\right) \in \mathbb{U}^{m \times m}$ and the $t$ th row of $\mathbf{V}^{k}\left(\mathbf{V}^{2 k+1}\right)^{*}=: \check{\mathbf{V}}=\left(\check{v}_{i j}\right) \epsilon$ $\mathbb{\natural}^{m \times m}$, respectively, for all $s, t=\overline{1, m} ; r=\operatorname{rank} \mathbf{V}^{k+1}=$ $\operatorname{rank} \mathbf{V}^{k}$.

The point (c) of Lemma 8 due to [23] has been generalized to $\mathbb{H}$ in [33]. Using this proposition, we have obtained the following two determinantal representations of the $W$ weighted Drazin inverse.

Theorem 9 (see [29]). Let $\mathbf{A} \in \mathbb{M}^{m \times n}$ and $\mathbf{W} \in \mathbb{M}_{r_{1}}^{n \times m}$ with $k=\operatorname{Ind}(\mathbf{A W})$ and $r=\operatorname{rank}(\mathbf{A W})^{k+1}=\operatorname{rank}(\mathbf{A W})^{k}$. Then the $W$-weighted Drazin inverse of $\mathbf{A}$ with respect to $\mathbf{W}$ possesses the following determinantal representations: 


$$
\begin{aligned}
& a_{i j}^{d, \mathbf{W}}=\frac{\sum_{t=1}^{m} \sum_{\alpha \in I_{r, m}\{t\}} \operatorname{rdet}_{t}\left(\left(\mathbf{V}^{2 k+1}\left(\mathbf{V}^{2 k+1}\right)^{*}\right)_{t}\left(\check{\mathbf{v}}_{i \cdot}\right)\right)_{\alpha}^{\alpha} \sum_{\alpha \in I_{r_{1}, n}\{j\}} \operatorname{rdet}_{j}\left(\left(\mathbf{W W}^{*}\right)_{j}\left(\check{\mathbf{w}}_{t \cdot}\right)\right)_{\alpha}^{\alpha}}{\sum_{\alpha \in I_{r, m}}\left|\left(\mathbf{V}^{2 k+1}\left(\mathbf{V}^{2 k+1}\right)^{*}\right)_{\alpha}^{\alpha}\right| \sum_{\alpha \in I_{r_{1}, n}}\left|\left(\mathbf{W}^{*}\right)_{\alpha}^{\alpha}\right|}, \\
& a_{i j}^{d, \mathbf{W}}=\frac{\sum_{t=1}^{n} \sum_{\beta \in J_{r_{1}, m}\{i\}} \operatorname{cdet}_{i}\left(\left(\mathbf{W}^{*} \mathbf{W}\right)_{\cdot t}\left(\widehat{\mathbf{w}}_{\cdot t}\right)\right)_{\beta}^{\beta} \sum_{\beta \in J_{r, n}\{t\}} \operatorname{cdet}_{t}\left(\left(\left(\mathbf{U}^{2 k+1}\right)^{*} \mathbf{U}^{2 k+1}\right)_{\cdot t}\left(\widehat{\mathbf{u}}_{\cdot j}\right)\right)_{\beta}^{\beta}}{\sum_{\beta \in J_{r_{1}, m}}\left|\left(\mathbf{W}^{*} \mathbf{W}\right)_{\beta}^{\beta}\right| \sum_{\beta \in J_{r, n}}\left|\left(\left(\mathbf{U}^{2 k+1}\right)^{*} \mathbf{U}^{2 k+1}\right)_{\beta}^{\beta}\right|},
\end{aligned}
$$

where $\check{\mathbf{V}}=\mathbf{V}^{k}\left(\mathbf{V}^{2 k+1}\right)^{*}, \check{\mathbf{W}}=\mathbf{V}^{k} \mathbf{W}^{*}$ and $\widehat{\mathbf{U}}=\left(\mathbf{U}^{2 k+1}\right)^{*} \mathbf{U}^{k}$, $\widehat{\mathbf{W}}=\mathbf{W}^{*} \mathbf{U}^{k}$.

In the special cases, when AW $\in \mathbb{H}^{m \times m}$ and WA $\epsilon$ $\mathbb{H}^{n \times n}$ are Hermitian, we can obtain simpler determinantal representations of the $W$-weighted Drazin inverse.

Theorem 10 (see [29]). If $\mathbf{A} \in \mathbb{H}^{m \times n}, \mathbf{W} \in \mathbb{T}^{n \times m}$, and $\mathbf{A W} \in$ $\mathbb{H}^{m \times m}$ are Hermitian with $k=\max \{\operatorname{Ind}(\mathbf{A W})$, Ind(WA) $\}$ and $\operatorname{rank}(\mathbf{A W})^{k+1}=\operatorname{rank}(\mathbf{A W})^{k}=r$, then the $W$-weighted Drazin inverse $\mathbf{A}_{d, W}=\left(a_{i j}^{d, W}\right) \in \mathbb{H}^{m \times n}$ with respect to $\mathbf{W}$ possesses the following determinantal representations:

$$
a_{i j}^{d, W}=\frac{\sum_{\beta \in J_{r, m}\{i\}} \operatorname{cdet}_{i}\left((\mathbf{A W})_{\cdot i}^{k+2}\left(\overline{\mathbf{v}}_{\cdot j}\right)\right)_{\beta}^{\beta}}{\sum_{\beta \in J_{r, m}}\left|\left((\mathbf{A W})^{k+2}\right)_{\beta}^{\beta}\right|}
$$

where $\overline{\mathbf{v}}_{. j}$ is the $j$ th column of $\overline{\mathbf{V}}=(\mathbf{A W})^{k} \mathbf{A}$ for all $j=\overline{1, m}$.

Theorem 11 (see [29]). If $\mathbf{A} \in \mathbb{H}^{m \times n}, \mathbf{W} \in \mathbb{H}^{n \times m}$, and $\mathbf{W A} \in$ $\mathbb{M}^{n \times n}$ are Hermitian with $k=\max \{\operatorname{Ind}(\mathbf{A W})$, Ind(WA) $\}$ and $\operatorname{rank}(\mathbf{W A})^{k+1}=\operatorname{rank}(\mathbf{W A})^{k}=r$, then the $W$-weighted Drazin inverse $\mathbf{A}_{d, W}=\left(a_{i j}^{d, W}\right) \in \mathbb{T}^{m \times n}$ with respect to $\mathbf{W}$ possesses the following determinantal representations:

$$
a_{i j}^{d, W}=\frac{\sum_{\alpha \in I_{r, n}\{j\}} \operatorname{rdet}_{j}\left((\mathbf{W A})_{j .}^{k+2}\left(\overline{\mathbf{u}}_{i \cdot}^{(k)}\right)\right)_{\alpha}^{\alpha}}{\sum_{\alpha \in I_{r, n}}\left|\left((\mathbf{W A})^{k+2}\right)_{\alpha}^{\alpha}\right|},
$$

where $\overline{\mathbf{u}}_{i}$. is the ith row of $\overline{\mathbf{U}}=\mathbf{A}(\mathbf{W A})^{k}$ for all $i=\overline{1, n}$.

\section{Cramer's Rule for the $W$-Weighted Drazin Inverse Solution}

3.1. Background of the Problem. In [27] Wei has established Cramer's rule for solving of a general restricted equation:

$$
\mathbf{W A W x}=\mathbf{b}, \quad \mathbf{x} \in \mathscr{R}\left[(\mathbf{A W})^{k_{1}}\right]
$$

where $\mathbf{A} \in \mathbb{C}^{m \times n}$ and $\mathbf{W} \in \mathbb{C}^{n \times m}$ with $\operatorname{Ind}(\mathbf{A W})=$ $k_{1}$, Ind (WA $)=k_{2}$ and $\operatorname{rank}(\mathbf{A W})^{k_{1}}=r_{1}, \operatorname{rank}(\mathbf{W A})^{k_{2}}=r_{2}$. He proved if $\mathbf{b} \in \mathscr{R}\left[(\mathbf{W})^{k_{2}} \mathbf{A}\right]$ and $r_{1}=r_{2}$, then (30) has a unique solution, $\mathbf{x}=\mathbf{A}_{d, W} \mathbf{b}$, which can be presented by the following Cramer rule:

$$
x_{j}=\frac{\operatorname{det}\left(\begin{array}{cc}
\mathbf{W A W}(j \rightarrow \mathbf{b}) & \mathbf{U}_{1} \\
\mathbf{V}_{1}(j \rightarrow 0) & 0
\end{array}\right)}{\operatorname{det}\left(\begin{array}{cc}
\mathbf{W A W} & \mathbf{U}_{1} \\
\mathbf{V}_{1} & 0
\end{array}\right)},
$$

where $\mathbf{U}_{1} \in \mathbb{C}_{n-r_{2}}^{n \times n-r_{2}}$ and $\mathbf{V}_{1}^{*} \in \mathbb{C}_{m-r_{1}}^{m \times m-r_{1}}$ are matrices whose columns form bases for $\mathcal{N}\left((\mathbf{W A})^{k_{2}}\right)$ and $\mathcal{N}\left((\mathbf{A W})^{k_{1}^{*}}\right)$, respectively.

Recently, within the framework of a theory of the column and row determinants, Song [28] has considered the characterization of the $W$-weighted Drazin inverse over the quaternion skew and presented a Cramer rule of the restricted matrix equation:

$$
\begin{aligned}
\mathbf{W}_{1} \mathbf{A W}_{1} \mathbf{X W}_{2} \mathbf{B} \mathbf{W}_{2}=\mathbf{D}, \\
\mathscr{R}_{r}(\mathbf{X}) \subset \mathscr{R}_{r}\left(\left(\mathbf{A} \mathbf{W}_{1}\right)^{k_{1}}\right), \\
\mathcal{N}_{r}(\mathbf{X}) \supset \mathcal{N}_{r}\left(\left(\mathbf{W}_{2} \mathbf{B}\right)^{k_{2}}\right), \\
\mathscr{R}_{l}(\mathbf{X}) \subset \mathscr{R}_{l}\left(\left(\mathbf{B W}_{2}\right)^{k_{2}}\right), \\
\mathscr{N}_{l}(\mathbf{X}) \supset \mathcal{N}_{l}\left(\left(\mathbf{W}_{1} \mathbf{A}\right)^{k_{1}}\right),
\end{aligned}
$$

where $\mathbf{A} \in \mathbb{H}^{m \times n}, \mathbf{W}_{1} \in \mathbb{H}^{n \times m}, \mathbf{B} \in \mathbb{H}^{p \times q}, \mathbf{W}_{2} \in \mathbb{H}^{q \times p}$, and $\mathbf{D} \in \mathbb{H}^{n \times p}$ with $k_{1}=\max \left\{\operatorname{Ind}\left(\mathbf{A} \mathbf{W}_{1}\right)\right.$, $\left.\operatorname{Ind}\left(\mathbf{W}_{1} \mathbf{A}\right)\right\}$, $k_{2}=\max \left\{\operatorname{Ind}\left(\mathbf{B W}_{2}\right)\right.$, Ind $\left.\left(\mathbf{W}_{2} \mathbf{B}\right)\right\}$, and $\operatorname{rank}\left(\mathbf{A W}_{1}\right)^{k_{1}}=s_{1}$, $\operatorname{rank}\left(\mathbf{B W}_{2}\right)^{k_{2}}=s_{2}$.

He proved that if

$$
\begin{aligned}
& \mathscr{R}_{r}(\mathbf{D}) \in \mathscr{R}_{r}\left(\left(\mathbf{W}_{1} \mathbf{A}\right)^{k_{1}},\left(\mathbf{W}_{2} \mathbf{B}\right)^{k_{2}}\right), \\
& \mathscr{R}_{l}(\mathbf{D}) \in \mathscr{R}_{l}\left(\left(\mathbf{A W}_{1}\right)^{k_{1}},\left(\mathbf{B W}_{2}\right)^{k_{2}}\right)
\end{aligned}
$$

and there exist auxiliary matrices of full column rank, $\mathbf{L}_{1} \in$ $\mathbb{H}_{n-s_{1}}^{n \times n-s_{1}}, \mathbf{M}_{1}^{*} \in \mathbb{H}_{m-s_{1}}^{m \times m-s_{1}}, \mathbf{L}_{2} \in \mathbb{T}_{q-s_{2}}^{q \times q-s_{2}}$, and $\mathbf{M}_{2}^{*} \in \mathbb{T}_{p-s_{2}}^{p \times p-s_{2}}$ with additional terms of their ranges and null spaces, then the restricted matrix equation (32) has a unique solution:

$$
\mathbf{X}=\mathbf{A}_{d, \mathbf{w}_{1}} \mathbf{D B}_{d, \mathbf{w}_{2}} \text {. }
$$

Using auxiliary matrices, $\mathbf{L}_{1}, \mathbf{M}_{1}, \mathbf{L}_{2}$, and $\mathbf{M}_{2}$, Song presented its Cramer's rule by analogy to (31). 
In this paper we have avoided such approach and have obtained explicit formulas for determinantal representations of the $W$-weighted Drazin inverse solutions of matrix equations by using only given matrices.

3.2. Cramer's Rules for the W-Weighted Drazin Inverse Solutions of Some Matrix Equations. Consider the matrix equation (32) with constraints (33). Denote ADB $=: \widetilde{\mathbf{D}}=\left(\widetilde{d}_{l f}\right) \epsilon$ $\mathbb{Q}^{m \times q}$ and $\overline{\mathbf{V}} \mathbf{D} \overline{\mathbf{U}}=: \overline{\mathbf{D}}=\left(\bar{d}_{l f}\right) \in \mathbb{U}^{m \times q}$, where $\overline{\mathbf{V}}:=\left(\mathbf{A W}_{1}\right)^{k_{1}} \mathbf{A}$ and $\overline{\mathbf{U}}:=\mathbf{B}\left(\mathbf{W}_{2} \mathbf{B}\right)^{k_{2}}$.

Theorem 12. Suppose that $\mathbf{D} \in \mathbb{T}^{n \times p}, \mathbf{A} \in \mathbb{T}^{m \times n}$, and $\mathbf{W}_{1} \in \mathbb{U}_{r_{1}}^{n \times m}$ with $k_{1}=\max \left\{\operatorname{Ind}\left(\mathbf{A W}_{1}\right), \operatorname{Ind}\left(\mathbf{W}_{1} \mathbf{A}\right)\right\}$, where $\operatorname{rank}\left(\mathbf{A W}_{1}\right)^{k_{1}}=s_{1}$, and $\mathbf{B} \in \mathbb{\mathbb { A } ^ { p } \times q}, \mathbf{W}_{2} \in \mathbb{T}_{r_{2}}^{q \times p}$ with $k_{2}=$ $\max \left\{\operatorname{Ind}\left(\mathbf{B W}_{2}\right), \operatorname{Ind}\left(\mathbf{W}_{2} \mathbf{B}\right)\right\}, \operatorname{rank}\left(\mathbf{B W}_{2}\right)^{k_{2}}=s_{2}$. If $\mathscr{R}_{r}(\mathbf{D}) \epsilon$ $\mathscr{R}_{r}\left(\left(\mathbf{W}_{1} \mathbf{A}\right)^{k_{1}},\left(\mathbf{W}_{2} \mathbf{B}\right)^{k_{2}}\right), \mathscr{R}_{l}(\mathbf{D}) \in \mathscr{R}_{l}\left(\left(\mathbf{A W}_{1}\right)^{k_{1}},\left(\mathbf{B W}_{2}\right)^{k_{2}}\right)$, then the restricted matrix equation (32) has a unique solution:

$$
\mathbf{X}=\mathbf{A}_{d, \mathbf{W}_{1}} \mathbf{D B}_{d, \mathbf{w}_{2}},
$$

which possesses the following determinantal representations for all $i=\overline{1, m}$ and $j=\overline{1, q}$.

(i) Consider

$$
x_{i j}=\sum_{l=1}^{m} \sum_{f=1}^{q}\left(v_{i l}^{D}\right)^{(2)} \tilde{d}_{l f}\left(u_{f j}^{D}\right)^{(2)},
$$

where $\left(v_{i l}^{D}\right)=\mathbf{V}^{D}$ is the Drazin inverse of $\mathbf{V}=\mathbf{A W}_{1}$ and $\left(v_{i l}^{D}\right)^{(2)}$ can be obtained by (23) and $\left(u_{f j}^{D}\right)=\mathbf{U}^{D}$ is the Drazin inverse of $\mathbf{U}=\mathbf{W}_{2} \mathbf{B}$ and $\left(u_{q j}^{D}\right)^{(2)}$ can be obtained by (21).

(ii) If $\mathbf{A W}_{1} \in \mathbb{G}^{m \times m}$ and $\mathbf{W}_{2} \mathbf{B} \in \mathbb{Q}^{q \times q}$ are Hermitian, then $x_{i j}$

$$
=\frac{\sum_{\beta \in J_{s_{1}, m}\{i\}} \operatorname{cdet}_{i}\left(\left(\mathbf{A W}_{1}\right)_{\cdot i}^{k_{1}+2}\left(\mathbf{d}_{\cdot j}^{\mathbf{B}}\right)\right)_{\beta}^{\beta}}{\sum_{\beta \in J_{s_{1}, m}}\left|\left(\left(\mathbf{A W}_{1}\right)^{k_{1}+2}\right)_{\beta}^{\beta}\right| \sum_{\alpha \in I_{s_{2}, q}}\left|\left(\left(\mathbf{W}_{2} \mathbf{B}\right)^{k_{2}+2}\right)_{\alpha}^{\alpha}\right|}
$$

or

$$
\begin{aligned}
& x_{i j} \\
& =\frac{\sum_{\alpha \in I_{s_{2}, q}\{j\}} \operatorname{rdet}_{j}\left(\left(\mathbf{W}_{2} \mathbf{B}\right)_{j .}^{k_{2}+2}\left(\mathbf{d}_{i \cdot}^{\mathbf{A}}\right)\right)_{\alpha}^{\alpha}}{\sum_{\beta \in J_{s_{1}, m}}\left|\left(\left(\mathbf{A W}_{1}\right)^{k_{1}+2}\right)_{\beta}^{\beta}\right| \sum_{\alpha \in I_{s_{2}, q}}\left|\left(\left(\mathbf{W}_{2} \mathbf{B}\right)^{k_{2}+2}\right)_{\alpha}^{\alpha}\right|},
\end{aligned}
$$

where

$$
\begin{array}{r}
\mathbf{d}_{\cdot j}^{\mathbf{B}}=\left(\sum_{\alpha \in I_{s_{2}, q}\{j\}} \operatorname{rdet}_{j}\left(\left(\mathbf{W}_{2} \mathbf{B}\right)_{j .}^{k_{2}+2}\left(\overline{\mathbf{d}}_{t}\right)\right)_{\alpha}^{\alpha}\right) \in \mathbb{\mathbb { Q } ^ { n \times 1 } ,} \\
t=\overline{1, n} \\
\mathbf{d}_{i \cdot}^{\mathbf{A}}=\left(\sum_{\beta \in J_{s_{1}, m}\{i\}} \operatorname{cdet}_{i}\left(\left(\mathbf{A W}_{1}\right)_{\cdot i}^{k_{1}+2}\left(\overline{\mathbf{d}}_{\cdot l}\right)\right)_{\beta}^{\beta}\right) \in \mathbb{Q}^{1 \times q}, \\
l=\overline{1, q}
\end{array}
$$

are the column vector and the row vector, respectively. $\overline{\mathbf{d}}_{i \text {. and }}$ $\overline{\mathbf{d}}_{. j}$ are the ith row and the $j$ th column of $\overline{\mathbf{D}}$ for all $i=\overline{1, n}$ and $j=\overline{1, p}$.

Proof. The existence and uniqueness of solution (36) can be proved similarly as in [28], Theorem 5.2.

(i) To derive a Cramer rule (37) we use the point (a) from Lemma 8. Then we obtain

$$
\mathbf{X}=\left(\left(\mathbf{A W}_{1}\right)^{D}\right)^{2} \operatorname{ADB}\left(\left(\mathbf{W}_{2} \mathbf{B}\right)^{D}\right)^{2}
$$

Denote ADB $=: \widetilde{\mathbf{D}}=\left(\widetilde{d}_{l f}\right) \in \mathbb{Q}^{m \times q}, \mathbf{V}:=\mathbf{A} \mathbf{W}_{1}$, and $\mathbf{U}:=$ $\mathbf{W}_{2} \mathbf{B}$. Then (42) will be written componentwise as follows:

$$
\begin{aligned}
x_{i j} & =\sum_{s=1}^{p} \sum_{t=1}^{n}\left(a_{i t}^{d, W_{1}}\right) d_{t s}\left(b_{s j}^{d, W_{2}}\right) \\
& =\sum_{s=1}^{p} \sum_{t=1}^{n}\left(\sum_{l=1}^{m}\left(v_{i l}^{D}\right)^{(2)} a_{l t}\right) d_{t s}\left(\sum_{f=1}^{q} b_{s f}\left(u_{f j}^{D}\right)^{(2)}\right) .
\end{aligned}
$$

By changing the order of summation, from here it follows (37).

(ii) If $\mathbf{A} \in \mathbb{G}_{r_{1}}^{m \times n}$ and $\mathbf{B} \in \mathbb{T}_{r_{2}}^{p \times q}$ and $\mathbf{A W}_{1} \in \mathbb{Q}^{m \times m}$ and $\mathbf{W}_{2} \mathbf{B} \in \mathbb{E}^{q \times q}$ are Hermitian, then by Theorems 10 and 11 the $W$-weighted Drazin inverses $\mathbf{A}_{d, W_{1}}=\left(a_{i j}^{d, W_{1}}\right) \in \mathbb{Q}^{m \times n}$ and $\mathbf{B}_{d, W_{2}}=\left(b_{i j}^{d, W_{2}}\right) \in \mathbb{G} \mathbb{G}^{q \times p}$ possess the following determinantal representations, respectively:

$$
a_{i j}^{d, W_{1}}=\frac{\sum_{\beta \in J_{s_{1}, m}\{i\}} \operatorname{cdet}_{i}\left(\left(\mathbf{A W}_{1}\right)_{\cdot i}^{k_{1}+2}\left(\overline{\mathbf{v}}_{\cdot j}\right)\right)_{\beta}^{\beta}}{\sum_{\beta \in J_{r, m}}\left|\left(\left(\mathbf{A W}_{1}\right)^{k_{1}+2}\right)_{\beta}^{\beta}\right|},
$$

where $\overline{\mathbf{v}}_{. j}$ is the $j$ th column of $\overline{\mathbf{V}}=\left(\mathbf{A W}_{\mathbf{1}}\right)^{k_{1}} \mathbf{A}$ for all $j=\overline{1, m}$ and

$$
b_{i j}^{d, W_{2}}=\frac{\sum_{\alpha \in I_{s_{2}, q}\{j\}} \operatorname{rdet}_{j}\left(\left(\mathbf{W}_{2} \mathbf{B}\right)_{j}^{k_{2}+2}\left(\overline{\mathbf{u}}_{i \cdot}\right)\right)_{\alpha}^{\alpha}}{\sum_{\alpha \in I_{s_{2}, q}}\left|\left(\left(\mathbf{W}_{2} \mathbf{B}\right)^{k_{2}+2}\right)_{\alpha}^{\alpha}\right|},
$$

where $\overline{\mathbf{u}}_{i}$. is the $i$ th row of $\overline{\mathbf{U}}=\mathbf{B}\left(\mathbf{W}_{2} \mathbf{B}\right)^{k_{2}}$ for all $i=\overline{1, p}$.

By componentwise writing of (36) we obtain

$$
x_{i j}=\sum_{s=1}^{p}\left(\sum_{t=1}^{n} a_{i t}^{d, W_{1}} d_{t s}\right) \cdot b_{s j}^{d, W_{2}} .
$$


Denote by $\widehat{\mathbf{d}_{s}}$ the $s$ th column of $\overline{\mathbf{V}} \mathbf{D}=\left(\mathbf{A W}_{1}\right)^{k_{1}} \mathbf{A D}=$ : $\widehat{\mathbf{D}}=\left(\widehat{d}_{i j}\right) \in \mathbb{H}^{m \times p}$ for all $s=\overline{1, p}$. It follows from $\sum_{t} \overline{\mathbf{v}}_{t} d_{t s}=$ $\widehat{\mathbf{d}_{\cdot s}}$ that

$$
\begin{aligned}
& \sum_{t=1}^{n} a_{i t}^{d, W_{1}} d_{t s} \\
& \quad=\sum_{t=1}^{n} \frac{\sum_{\beta \in J_{s_{1}, m}\{i\}} \operatorname{cdet}_{i}\left(\left(\mathbf{A W}_{1}\right)_{\cdot i}^{k_{1}+2}\left(\overline{\mathbf{v}}_{\cdot t}\right)\right)_{\beta}^{\beta}}{\sum_{\beta \in J_{s_{1}, m}}\left|\left(\left(\mathbf{A W}_{1}\right)^{k_{1}+2}\right)_{\beta}^{\beta}\right|} \cdot d_{t s}
\end{aligned}
$$

$$
\begin{aligned}
& =\frac{\sum_{\beta \in J_{s_{1}, m}\{i\}} \sum_{t=1}^{n} \operatorname{cdet}_{i}\left(\left(\mathbf{A W}_{1}\right)_{\cdot i}^{k_{1}+2}\left(\overline{\mathbf{v}}_{\cdot t}\right)\right)_{\beta}^{\beta} \cdot d_{t s}}{\sum_{\beta \in J_{s_{1}, m}}\left|\left(\left(\mathbf{A W}_{1}\right)^{k_{1}+2}\right)_{\beta}^{\beta}\right|} \\
& =\frac{\sum_{\beta \in J_{s_{1}, m}\{i\}} \operatorname{cdet}_{i}\left(\left(\mathbf{A W}_{1}\right)_{\cdot i}^{k_{1}+2}\left(\hat{\mathbf{d}}_{\cdot s}\right)\right)_{\beta}^{\beta}}{\sum_{\beta \in J_{s_{1}, m}}\left|\left(\left(\mathbf{A W}_{1}\right)^{k_{1}+2}\right)_{\beta}^{\beta}\right|} .
\end{aligned}
$$

Suppose that $\mathbf{e}_{s \text {. }}$ and $\mathbf{e}_{\cdot s}$ are, respectively, the unit row vector and the unit column vector whose components are 0 , except the sth components, which are 1. Substituting (47) and (45) into (46), we obtain

$$
x_{i j}=\sum_{s=1}^{p} \frac{\sum_{\beta \in J_{s_{1}, m}\{i\}} \operatorname{cdet}_{i}\left(\left(\mathbf{A W}_{1}\right)_{\cdot i}^{k_{1}+2}\left(\widehat{\mathbf{d}}_{\cdot s}\right)\right)_{\beta}^{\beta}}{\sum_{\beta \in J_{s_{1}, m}}\left|\left(\left(\mathbf{A W}_{1}\right)^{k_{1}+2}\right)_{\beta}^{\beta}\right|} \frac{\sum_{\alpha \in I_{s_{2}, q}\{j\}} \operatorname{rdet}_{j}\left(\left(\mathbf{W}_{2} \mathbf{B}\right)_{j .}^{k_{2}+2}\left(\overline{\mathbf{u}}_{s \cdot}\right)\right)_{\alpha}^{\alpha}}{\sum_{\alpha \in I_{s_{2}, q}}\left|\left(\left(\mathbf{W}_{2} \mathbf{B}\right)^{k_{2}+2}\right)_{\alpha}^{\alpha}\right|} .
$$

Since

$$
\begin{aligned}
\widehat{\mathbf{d}}_{\cdot s} & =\sum_{t=1}^{n} \mathbf{e}_{\cdot t}{\widehat{d_{t s}}}, \\
\overline{\mathbf{u}}_{s \cdot} & =\sum_{l=1}^{q} \bar{u}_{s l} \mathbf{e}_{l \cdot}, \\
\sum_{s=1}^{p}{\widehat{d_{t s}}}_{\bar{u}_{s l}} & =\bar{d}_{t l},
\end{aligned}
$$

$$
\begin{aligned}
x_{i j}=\frac{\sum_{s=1}^{p} \sum_{t=1}^{n} \sum_{l=1}^{q} \sum_{\beta \in J_{s_{1}, m}\{i\}} \operatorname{cdet}_{i}\left(\left(\mathbf{A W}_{1}\right)_{\cdot i}^{k_{1}+2}\left(\mathbf{e}_{\cdot t}\right)\right)_{\beta}^{\beta}{\widehat{d_{t s}}}_{\bar{u}_{s l}} \sum_{\alpha \in I_{s_{2}, q}\{j\}} \operatorname{rdet}_{j}\left(\left(\mathbf{W}_{2} \mathbf{B}\right)_{j .}^{k_{2}+2}\left(\mathbf{e}_{l .}\right)\right)_{\alpha}^{\alpha}}{\sum_{\beta \in J_{s_{1}, m}}\left|\left(\left(\mathbf{A W}_{1}\right)^{k_{1}+2}\right)_{\beta}^{\beta}\right| \sum_{\alpha \in I_{s_{2}, q}}\left|\left(\left(\mathbf{W}_{2} \mathbf{B}\right)^{k_{2}+2}\right)_{\alpha}^{\alpha}\right|} \\
=\frac{\sum_{t=1}^{n} \sum_{l=1}^{q} \sum_{\beta \in J_{s_{1}, m}\{i\}} \operatorname{cdet}_{i}\left(\left(\mathbf{A W}_{1}\right)_{\cdot i}^{k_{1}+2}\left(\mathbf{e}_{\cdot t}\right)\right)_{\beta}^{\beta} \bar{d}_{t l} \sum_{\alpha \in I_{s_{2}, q}\{j\}} \operatorname{rdet}_{j}\left(\left(\mathbf{W}_{2} \mathbf{B}\right)_{j .}^{k_{2}+2}\left(\mathbf{e}_{l \cdot}\right)\right)_{\alpha}^{\alpha}}{\sum_{\beta \in J_{s_{1}, m}}\left|\left(\left(\mathbf{A W}_{1}\right)^{k_{1}+2}\right)_{\beta}^{\beta}\right| \sum_{\alpha \in I_{s_{2}, q}}\left|\left(\left(\mathbf{W}_{2} \mathbf{B}\right)^{k_{2}+2}\right)_{\alpha}^{\alpha}\right|} .
\end{aligned}
$$

Denote by

$$
\begin{aligned}
d_{i l}^{\mathbf{A}} & :=\sum_{\beta \in J_{s_{1}, m}\{i\}} \operatorname{cdet}_{i}\left(\left(\mathbf{A W}_{1}\right)_{\cdot i}^{k_{1}+2}\left(\overline{\mathbf{d}}_{\cdot l}\right)\right)_{\beta}^{\beta} \\
& =\sum_{t=1}^{n} \sum_{\beta \in J_{s_{1}, m}\{i\}} \operatorname{cdet}_{i}\left(\left(\mathbf{A W}_{1}\right)_{\cdot i}^{k_{1}+2}\left(\mathbf{e}_{\cdot t}\right)\right)_{\beta}^{\beta} \bar{d}_{t l}
\end{aligned}
$$

the $l$ th component of a row vector $\mathbf{d}_{i .}^{\mathbf{A}}=\left(d_{i 1}^{\mathbf{A}}, \ldots, d_{i q}^{\mathbf{A}}\right)$ for all $l=\overline{1, q}$. Substituting it into $(50)$, we have

$$
=\frac{\sum_{l=1}^{q} d_{i l}^{\mathbf{A}} \sum_{\alpha \in I_{s_{2}, q}\{j\}} \operatorname{rdet}_{j}\left(\left(\mathbf{W}_{2} \mathbf{B}\right)_{j .}^{k_{2}+2}\left(\mathbf{e}_{l \cdot}\right)\right)_{\alpha}^{\alpha}}{\sum_{\beta \in J_{s_{1}, m}}\left|\left(\left(\mathbf{A W}_{1}\right)^{k_{1}+2}\right)_{\beta}^{\beta}\right| \sum_{\alpha \in I_{s_{2}, q}}\left|\left(\left(\mathbf{W}_{2} \mathbf{B}\right)^{k_{2}+2}\right)_{\alpha}^{\alpha}\right|} .
$$
Since $\sum_{l=1}^{q} d_{i l}^{\mathbf{A}} \mathbf{e}_{l .}=\mathbf{d}_{i}^{\mathbf{A}}$, then it follows (39). 
If we denote by

$$
\begin{aligned}
d_{t j}^{\mathbf{B}} & :=\sum_{l=1}^{q} \bar{d}_{t l} \sum_{\alpha \in I_{s_{2}, q}\{j\}} \operatorname{rdet}_{j}\left(\left(\mathbf{W}_{2} \mathbf{B}\right)_{j .}^{k_{2}+2}\left(\mathbf{e}_{l \cdot}\right)\right)_{\alpha}^{\alpha} \\
& =\sum_{\alpha \in I_{s_{2}, q}\{j\}} \operatorname{rdet}_{j}\left(\left(\mathbf{W}_{2} \mathbf{B}\right)_{j .}^{k_{2}+2}\left(\overline{\mathbf{d}}_{t \cdot}\right)\right)_{\alpha}^{\alpha}
\end{aligned}
$$

the $t$ th component of a column vector $\mathbf{d}_{\cdot j}^{\mathbf{B}}=\left(d_{1 j}^{\mathbf{B}}, \ldots, d_{n j}^{\mathbf{B}}\right)^{T}$ for all $t=\overline{1, n}$ and substituting it into (50), we obtain

$$
x_{i j}=\frac{\sum_{t=1}^{n} \sum_{\beta \in J_{s_{1}, m}\{i\}} \operatorname{cdet}_{i}\left(\left(\mathbf{A W}_{1}\right)_{\cdot i}^{k_{1}+2}\left(\mathbf{e}_{\cdot t}\right)\right)_{\beta}^{\beta} d_{t j}^{\mathbf{B}}}{\sum_{\beta \in J_{r_{1}, n}}\left|\left(\mathbf{A}^{*} \mathbf{A}\right)_{\beta}^{\beta}\right| \sum_{\alpha \in I_{r_{2}, p}}\left|\left(\mathbf{B B}^{*}\right)_{\alpha}^{\alpha}\right|} .
$$

Since $\sum_{t=1}^{n} \mathbf{e}_{\cdot t} d_{t j}^{\mathbf{B}}=\mathbf{d}_{\cdot j}^{\mathbf{B}}$, then it follows (38).

Remark 13. To establish a Cramer rule of (32) we will not use the determinantal representations (28) and (28) for (36) because the corresponding determinantal representations of its solution will be too cumbersome. But they are suitable in the following corollaries.

Corollary 14. Suppose that the following restricted matrix equation is given:

$$
\begin{aligned}
& \text { WAWX }=\mathbf{D}, \\
& \mathscr{R}_{r}(\mathbf{X}) \subset \mathscr{R}_{r}\left((\mathbf{A W})^{k}\right), \\
& \mathcal{N}_{l}(\mathbf{X}) \supset \mathscr{N}_{l}\left((\mathbf{W A})^{k}\right),
\end{aligned}
$$

where $\mathbf{A} \in \mathbb{T}^{m \times n}$ and $\mathbf{W} \in \mathbb{H}_{r_{1}}^{n \times m}$ with $k=\max \{\operatorname{Ind}(\mathbf{A W})$, $\operatorname{Ind}(\mathbf{W A})\}$ and $\mathbf{D} \in \mathbb{T}^{n \times p}$. If $\mathscr{R}_{r}(\mathbf{D}) \subset \mathscr{R}_{r}\left((\mathbf{A W})^{k}\right)$ and $\mathscr{N}_{l}(\mathrm{D}) \supset \mathcal{N}_{l}\left((\mathbf{W A})^{k}\right)$, then the restricted matrix equation (55)(56) has a unique solution:

$$
\mathbf{X}=\mathbf{A}_{d, W} \mathbf{D}
$$

which possess the following determinantal representations for all $i=\overline{1, m}$ and $j=\overline{1, p}$.

(i) Consider

$$
x_{i j}=\frac{\sum_{t=1}^{n} \sum_{\beta \in J_{r_{1}, m}\{i\}} \operatorname{cdet}_{i}\left(\left(\mathbf{W}^{*} \mathbf{W}\right)_{\cdot t}\left(\widehat{\mathbf{W}}_{\cdot t}\right)\right)_{\beta}^{\beta} \sum_{\beta \in J_{r, n}\{t\}} \operatorname{cdet}_{t}\left(\left(\left(\mathbf{U}^{2 k+1}\right)^{*} \mathbf{U}^{2 k+1}\right)_{\cdot t}\left(\widehat{\mathbf{d}}_{\cdot j}\right)\right)_{\beta}^{\beta}}{\sum_{\beta \in J_{r_{1}, m}}\left|\left(\mathbf{W}^{*} \mathbf{W}\right)_{\beta}^{\beta}\right| \sum_{\beta \in J_{r, n}}\left|\left(\left(\mathbf{U}^{2 k+1}\right)^{*} \mathbf{U}^{2 k+1}\right)_{\beta}^{\beta}\right|}
$$

where $\mathbf{U}=\mathbf{W A}, \widehat{\mathbf{d}}_{\text {. }}$ is the $j$ th column of $\widehat{\mathbf{D}}=\widehat{\mathbf{U}} \mathbf{D}=$ $\left(\mathbf{U}^{2 k+1}\right)^{*} \mathbf{U}^{k} \mathbf{D}, \widehat{\mathbf{W}}=\mathbf{W}^{*} \mathbf{U}^{k}$, and $r=\operatorname{rank}(\mathbf{W A})^{k+1}=$ $\operatorname{rank}(\mathbf{W A})^{k}$.

(ii) Consider

$$
x_{i j}=\sum_{q=1}^{m}\left(v_{i q}^{D}\right)^{(2)} r_{q j}
$$

where $\left(v_{i q}^{D}\right)^{(2)}$ can be obtained by (23) and $\mathbf{A D}=\mathbf{R}=\left(r_{q j}\right) \epsilon$ $\mathbb{\mathbb { N } ^ { m \times p }}$. (iii) If $\mathbf{A W} \in \mathbb{H}^{m \times m}$ is Hermitian, then

$$
x_{i j}=\frac{\sum_{\beta \in J_{r, m}\{i\}} \operatorname{cdet}_{i}\left((\mathbf{A W})_{\cdot i}^{k+2}\left(\mathbf{f}_{\cdot j}\right)\right)_{\beta}^{\beta}}{\sum_{\beta \in J_{r, m}}\left|\left((\mathbf{A W})^{k+2}\right)_{\beta}^{\beta}\right|},
$$

where $\mathbf{f}_{. j}$ is the $j$ th column of $\mathbf{F}=\overline{\mathbf{V}} \mathbf{D}=(\mathbf{A W})^{k} \mathbf{A D}$.

Proof. To derive a Cramer rule (58), we use the determinantal representation (27) for $\mathbf{A}_{d, W}$. Then

$$
x_{i j}=\sum_{s=1}^{p} a_{i s}^{d, \mathbf{W}} d_{s j}=\sum_{s=1}^{p}\left[\frac{\sum_{t=1}^{n} \sum_{\beta \in J_{r_{1}, m}\{i\}} \operatorname{cdet}_{i}\left(\mathbf{W}^{*} \mathbf{W}\right)_{\cdot t}\left(\widehat{\mathbf{w}}_{\cdot t}\right)_{\beta}^{\beta} \sum_{\beta \in J_{r, n}\{t\}} \operatorname{cdet}_{t}\left(\left(\mathbf{U}^{2 k+1}\right)^{*} \mathbf{U}^{2 k+1}\right)_{\cdot t}\left(\widehat{\mathbf{u}}_{\cdot s}\right)_{\beta}^{\beta}}{\sum_{\beta \in J_{r_{1}, m}}\left|\left(\mathbf{W}^{*} \mathbf{W}\right)_{\beta}^{\beta}\right| \sum_{\beta \in J_{r, n}}\left|\left(\left(\mathbf{U}^{2 k+1}\right)^{*} \mathbf{U}^{2 k+1}\right)_{\beta}^{\beta}\right|}\right] d_{s j} .
$$

Denote $\widehat{\mathbf{D}}=\widehat{\mathbf{U}} \mathbf{D}=\left(\mathbf{U}^{2 k+1}\right)^{*} \mathbf{U}^{k} \mathbf{D}$, where $\widehat{\mathbf{D}}=\left(\widehat{d}_{s j}\right) \in \mathbb{\boxplus}^{n \times p}$. Since

$$
\sum_{s=1}^{p} \widehat{\mathbf{u}}_{\cdot s} d_{s j}=\widehat{\mathbf{d}}_{\cdot j}
$$

where $\widehat{\mathbf{d}}_{. j}$ is the $j$ th column of $\mathbf{D}$, then (58) follows from (61).

Cramer's rule (59) and (60) immediately follows from Theorem 12 by putting $\mathbf{W}_{1}=\mathbf{W}$ and $\mathbf{W}_{2} \mathbf{B}=\mathbf{I}$.

Remark 15. In the complex case, that is, $\mathbf{A} \in \mathbb{C}^{m \times n}, \mathbf{W} \in$ $\mathbb{C}_{r_{1}}^{n \times m}$, and $\mathbf{D} \in \mathbb{C}^{n \times p}$, we substitute usual determinants for all corresponding row and column determinants in (58), (59), and (60).

Note that in case (iii), the condition AW $\in \mathbb{C}^{m \times m}$ being Hermitian is not necessary; then in the complex case (60) will have the form

$$
x_{i j}=\frac{\sum_{\beta \in J_{r, m}\{i\}}\left|\left((\mathbf{A W})_{\cdot i}^{k+2}\left(\mathbf{f}_{\cdot j}\right)\right)_{\beta}^{\beta}\right|}{\sum_{\beta \in J_{r, m}}\left|\left((\mathbf{A W})^{k+2}\right)_{\beta}^{\beta}\right|},
$$

where $\mathbf{f}_{\cdot j}$ is the $j$ th column of $\mathbf{F}=\overline{\mathbf{V}} \mathbf{D}=(\mathbf{A W})^{k} \mathbf{A D}$. 
Corollary 16. Suppose that the following restricted matrix equation is given:

$$
\begin{aligned}
& \mathbf{X W B W}=\mathbf{D}, \\
& \mathscr{R}_{l}(\mathbf{X}) \subset \mathscr{R}_{l}\left((\mathbf{B W})^{k}\right), \\
& \mathcal{N}_{r}(\mathbf{X}) \supset \mathscr{N}_{r}\left((\mathbf{B A})^{k}\right),
\end{aligned}
$$

where $\mathbf{B} \in \mathbb{H}^{p \times q}$ and $\mathbf{W} \in \mathbb{H}_{r_{1}}^{q \times p}$ with $k=\max \{\operatorname{Ind}(\mathbf{A W})$, Ind $(\mathbf{W B})\}$ and $\mathbf{D} \in \mathbb{H}^{n \times p}$. If $\mathscr{R}_{l}(\mathbf{D}) \subset \mathscr{R}_{l}\left((\mathbf{B W})^{k}\right)$ and
$\mathcal{N}_{r}(\mathrm{D}) \supset \mathcal{N}_{r}\left((\mathbf{W B})^{k}\right)$, then the restricted matrix equation (64) has a unique solution:

$$
\mathbf{X}=\mathbf{D B}_{d, W}
$$

which possesses the following determinantal representations for $i=\overline{1, n}$ and $j=\overline{1, q}$.

(i) Consider

$$
x_{i j}=\frac{\sum_{l=1}^{p} \sum_{\alpha \in I_{r, p}\{l\}} \operatorname{rdet}_{l}\left(\left(\mathbf{V}^{2 k+1}\left(\mathbf{V}^{2 k+1}\right)^{*}\right)_{l .}\left(\check{\mathbf{d}}_{i \cdot}\right)\right)_{\alpha}^{\alpha} \sum_{\alpha \in I_{r_{1}, q}\{j\}} \operatorname{rdet}_{j}\left(\left(\mathbf{W W}^{*}\right)_{j}\left(\check{\mathbf{w}}_{l \cdot}\right)\right)_{\alpha}^{\alpha}}{\sum_{\alpha \in I_{r, m}}\left|\left(\mathbf{V}^{2 k+1}\left(\mathbf{V}^{2 k+1}\right)^{*}\right)_{\alpha}^{\alpha}\right| \sum_{\alpha \in I_{r_{1}, n}}\left|\left(\mathbf{W} \mathbf{W}^{*}\right)_{\alpha}^{\alpha}\right|},
$$

where $\mathbf{V}=\mathbf{B W}, \check{\mathbf{d}}_{i}$. is the ith row of $\check{\mathbf{D}}=\mathbf{D} \check{\mathbf{V}}=\mathbf{D V}^{k}\left(\mathbf{V}^{2 k+1}\right)^{*}$, $\check{\mathbf{w}}_{l}$. is the lth row of $\check{\mathbf{W}}=\mathbf{V}^{k} \mathbf{W}^{*}$, and $r=\operatorname{rank}(\mathbf{B W})^{k+1}=$ $\operatorname{rank}(\mathbf{B W})^{k}$.

(ii) Consider

$$
x_{i j}=\sum_{t=1}^{q} l_{i t}\left(u_{t j}^{D}\right)^{(2)} \text {, }
$$

where $\left(u_{t j}^{D}\right)^{(2)}$ can be obtained by (21) and $\mathbf{D B}=\mathbf{L}=\left(l_{i t}\right) \epsilon$ $\mathbb{n}^{n \times q}$.

(iii) If $\mathbf{W B} \in \mathbb{H}^{q \times q}$ is Hermitian, then

$$
x_{i j}=\frac{\sum_{\alpha \in I_{r, q}\{j\}} \operatorname{rdet}_{j}\left((\mathbf{W B})_{j .}^{k+2}\left(\mathbf{g}_{i \cdot}\right)\right)_{\alpha}^{\alpha}}{\sum_{\alpha \in I_{r, q}}\left|\left((\mathbf{W B})^{k+2}\right)_{\alpha}^{\alpha}\right|},
$$

where $\mathbf{g}_{i}$. is the ith row of $\mathbf{G}=\mathbf{D B}(\mathbf{W B})^{k}$ for all $i=\overline{1, n}$.

Proof. The proof is similar to the proof of Corollary 14 in the point (i) and follows from Theorem 12 by putting $\mathbf{W}_{2}=\mathbf{W}$ and $\mathbf{A W}_{1}=\mathbf{I}$.

Remark 17. In the complex case, that is, $\mathbf{B} \in \mathbb{C}^{p \times q}, \mathbf{W} \in$ $\mathbb{C}_{r_{1}}^{q \times p}$, and $\mathbf{D} \in \mathbb{C}^{n \times p}$, we substitute usual determinants for all corresponding row and column determinants in (66), (67), and (68). Herein the condition WB $\in \mathbb{C}^{n \times n}$ being Hermitian is not necessary; then in the complex case (68) can be represented as follows:

$$
x_{i j}=\frac{\sum_{\alpha \in I_{r, q}\{j\}}\left|\left((\mathbf{W B})_{j .}^{k+2}\left(\mathbf{g}_{i \cdot}\right)\right)_{\alpha}^{\alpha}\right|}{\sum_{\alpha \in I_{r, q}}\left|\left((\mathbf{W B})^{k+2}\right)_{\alpha}^{\alpha}\right|},
$$

where $\mathbf{g}_{i}$ is the $i$ th row of $\mathbf{G}=\mathbf{D B}(\mathbf{W B})^{k}$ for all $i=\overline{1, n}$.

\section{Examples}

In this section, we give examples to illustrate our results.

(1) Let us consider the matrix equation

$$
\text { WAWX }=\text { D }
$$

with the restricted conditions (56), where

$$
\begin{aligned}
& \mathbf{A}=\left(\begin{array}{ccc}
0 & i & 0 \\
k & 1 & i \\
1 & 0 & 0 \\
1 & -k & -j
\end{array}\right), \\
& \mathbf{W}=\left(\begin{array}{cccc}
k & 0 & i & 0 \\
-j & k & 0 & 1 \\
0 & 1 & 0 & -k
\end{array}\right), \\
& \mathbf{D}=\left(\begin{array}{ccc}
k & i \\
i & -j \\
1 & -i
\end{array}\right) .
\end{aligned}
$$

Then

$$
\begin{aligned}
& \mathbf{V}=\mathbf{A W}=\left(\begin{array}{cccc}
-k & -j & 0 & i \\
-1-j & i+k & j & 1+j \\
k & 0 & i & 0 \\
-i+k & 1-j & i & i-k
\end{array}\right), \\
& \mathbf{U}=\mathbf{W A}=\left(\begin{array}{lll}
i & j & 0 \\
0 & k & 0 \\
0 & 0 & 0
\end{array}\right)
\end{aligned}
$$

and $\operatorname{rank} \mathbf{W}=3, \operatorname{rank} \mathbf{V}=3, \operatorname{rank} \mathbf{V}^{3}=\operatorname{rank} \mathbf{V}^{2}=2$, and $\operatorname{rank} \mathbf{U}^{2}=\operatorname{rank} \mathbf{U}=2$. So, Ind $\mathbf{V}=2$, Ind $\mathbf{U}=1$, and $k=$ $\max \{\operatorname{Ind}(\mathbf{A W}), \operatorname{Ind}(\mathbf{W A})\}=2$.

We will find the $W$-weighted Drazin inverse solution of (70) by its determinantal representation (58). We have

$$
\mathbf{U}^{2}=\left(\begin{array}{ccc}
-1 & i+k & 0 \\
0 & -1 & \\
0 & 0 & 0
\end{array}\right)
$$




$$
\begin{aligned}
& \mathbf{U}^{5}=\left(\begin{array}{ccc}
i & 2+3 j & 0 \\
0 & k & \\
0 & 0 & 0
\end{array}\right), \\
& \left(\mathbf{U}^{5}\right)^{*}=\left(\begin{array}{ccc}
-i & 0 & 0 \\
2-3 j & -k & \\
0 & 0 & 0
\end{array}\right), \\
& \left(\mathbf{U}^{5}\right)^{*} \mathbf{U}^{5}=\left(\begin{array}{ccc}
1 & -2 i-3 k & 0 \\
2 i+3 k & 14 & \\
0 & 0 & 0
\end{array}\right) \text {, } \\
& \widehat{\mathbf{D}}=\left(\mathbf{U}^{5}\right)^{*} \mathbf{U}^{2} \mathbf{D} \\
& =\left(\begin{array}{cc}
i-j-k & -j \\
1+3 i+6 j-2 k & 4 i-2 k \\
0 & 0
\end{array}\right),
\end{aligned}
$$$$
\mathbf{W}^{*}=\left(\begin{array}{ccc}
-k & j & 0 \\
0 & -k & 1 \\
-i & 0 & 0 \\
0 & 1 & k
\end{array}\right)
$$$$
\mathbf{W}^{*} \mathbf{W}=\left(\begin{array}{cccc}
2 & i & -j & j \\
-i & 2 & 0 & -2 k \\
j & 0 & 1 & 0 \\
-j & 2 k & 0 & 2
\end{array}\right) \text {, }
$$$$
\widehat{\mathbf{W}}=\mathbf{W}^{*} \mathbf{U}^{2}=\left(\begin{array}{ccc}
-k & 1-2 j & 0 \\
0 & i+k & 0 \\
i & 1+j & 0 \\
0 & -1 & 0
\end{array}\right) \text {. }
$$

Since by (58)

$$
x_{11}=\frac{\sum_{t=1}^{3} \sum_{\beta \in I_{3,4}\{1\}} \operatorname{cdet}_{1}\left(\left(\mathbf{W}^{*} \mathbf{W}\right)_{\cdot 1}\left(\widehat{\mathbf{w}}_{\cdot t}\right)\right)_{\beta}^{\beta} \sum_{\beta \in J_{2,3}\{t\}} \operatorname{cdet}_{t}\left(\left(\left(\mathbf{U}^{5}\right)^{*} \mathbf{U}^{5}\right)_{\cdot t}\left(\widehat{\mathbf{d}}_{\cdot 1}\right)\right)_{\beta}^{\beta}}{\sum_{\beta \in J_{3,4}}\left|\left(\mathbf{W}^{*} \mathbf{W}\right)_{\beta}^{\beta}\right| \sum_{\beta \in J_{2,3}}\left|\left(\left(\mathbf{U}^{5}\right)^{*} \mathbf{U}^{5}\right)_{\beta}^{\beta}\right|},
$$

where

$$
\begin{aligned}
& \sum_{\beta \in I_{3,4}\{1\}} \operatorname{cdet}_{1}\left(\left(\mathbf{W}^{*} \mathbf{W}\right)_{\cdot 1}\left(\widehat{\mathbf{w}}_{\cdot 1}\right)\right)_{\beta}^{\beta} \\
& =\operatorname{cdet}_{1}\left(\begin{array}{ccc}
k & i & -j \\
0 & 2 & 0 \\
i & 0 & 1
\end{array}\right)+\operatorname{cdet}_{1}\left(\begin{array}{ccc}
k & i & j \\
0 & 2 & -2 k \\
0 & 2 k & 1
\end{array}\right) \\
& +\operatorname{cdet}_{1}\left(\begin{array}{ccc}
k & -j & j \\
i & 1 & 0 \\
0 & 0 & 2
\end{array}\right)=0 \text {, } \\
& \sum_{\beta \in I_{3,4}\{1\}} \operatorname{cdet}_{1}\left(\left(\mathbf{W}^{*} \mathbf{W}\right)_{\cdot 1}\left(\widehat{\mathbf{w}}_{\cdot 2}\right)\right)_{\beta}^{\beta}=-2 j, \\
& \sum_{\beta \in I_{3,4}\{1\}} \operatorname{cdet}_{1}\left(\left(\mathbf{W}^{*} \mathbf{W}\right)_{\cdot 1}\left(\widehat{\mathbf{w}}_{\cdot 3}\right)\right)_{\beta}^{\beta}=0, \\
& \sum_{\beta \in J_{3,4}}\left|\left(\mathbf{W}^{*} \mathbf{W}\right)_{\beta}^{\beta}\right|=2 \text {, } \\
& \sum_{\beta \in J_{2,3}\{1\}} \operatorname{cdet}_{1}\left(\left(\left(\mathbf{U}^{5}\right)^{*} \mathbf{U}^{5}\right)_{\cdot 1}\left(\widehat{\mathbf{d}}_{\cdot 1}\right)\right)_{\beta}^{\beta} \\
& =\operatorname{cdet}_{1}\left(\begin{array}{cc}
i-j-k & -2 i-3 k \\
1+3 i+6 j-2 k & 14
\end{array}\right) \\
& +\operatorname{cdet}_{1}\left(\begin{array}{cc}
i-j-k & 0 \\
0 & 0
\end{array}\right)=-2 i-j-k \text {, }
\end{aligned}
$$

$$
\sum_{\beta \in \in_{2,3}\{2\}} \operatorname{cdet}_{2}\left(\left(\left(\mathbf{U}^{5}\right)^{*} \mathbf{U}^{5}\right)_{\cdot 2}\left(\widehat{\mathbf{d}}_{\cdot 1}\right)\right)_{\beta}^{\beta}=j,
$$$$
\sum_{\beta \in J_{2,3}\{3\}} \operatorname{cdet}_{3}\left(\left(\left(\mathbf{U}^{5}\right)^{*} \mathbf{U}^{5}\right)_{\cdot 3}\left(\widehat{\mathbf{d}}_{11}\right)\right)_{\beta}^{\beta}=0,
$$$$
\sum_{\beta \in J_{2,3}}\left|\left(\left(\mathbf{U}^{5}\right)^{*} \mathbf{U}^{5}\right)_{\beta}^{\beta}\right|=1 \text {, }
$$

then

$$
\begin{aligned}
x_{11} & =\frac{0 \cdot(-2 i-j-k)+(-2 j) \cdot j+0 \cdot 0}{2 \cdot 1}=1 . \\
x_{12} & =\frac{0 \cdot(-2+2 j)+(-2 j) \cdot i+0 \cdot 0}{2 \cdot 1}=k, \\
x_{21} & =\frac{2 j \cdot(-2 i-j-k)+(10 i-4 k) \cdot j+0 \cdot 0}{2 \cdot 1} \\
& =1+i+7 k, \\
x_{22} & =\frac{2 j \cdot(-2+2 j)+(10 i-4 k) \cdot i+0 \cdot 0}{2 \cdot 1} \\
& =-7-4 j, \\
x_{31} & =\frac{10 i \cdot(-2 i-j-k)+j \cdot j+0 \cdot 0}{2 \cdot 1}
\end{aligned}
$$




$$
\begin{aligned}
& =9.5+5 j-5 k, \\
x_{32} & =\frac{10 i \cdot(-2+2 j)+j \cdot i+0 \cdot 0}{2 \cdot 1}=-10 i+9.5 k,
\end{aligned}
$$

We finally get

$$
\mathbf{X}=\left(\begin{array}{cc}
1 & k \\
1+i+7 k & -7-4 j \\
9.5+5 j-5 k & -10 i+9.5 k
\end{array}\right)
$$

(2) Let now us consider the matrix equation

$$
\mathbf{W}_{1} \mathrm{AW}_{1} \mathbf{X W}_{2} \mathbf{B W}_{2}=\mathbf{D} \text {, }
$$

with constraints (33), where

$$
\begin{aligned}
& \mathbf{A}=\left(\begin{array}{cccc}
k & 0 & i & 0 \\
-j & k & 0 & 1 \\
0 & 1 & 0 & -k
\end{array}\right) \\
& \mathbf{W}_{1}=\left(\begin{array}{ccc}
k & -j & 0 \\
0 & k & 1 \\
i & 0 & 0 \\
0 & 1 & -k
\end{array}\right) \\
& \mathbf{W}_{2}=\left(\begin{array}{cc}
k & -i \\
j & 0 \\
0 & 1
\end{array}\right) \text {, } \\
& \mathbf{B}=\left(\begin{array}{lll}
k & j & 0 \\
j & 0 & 1
\end{array}\right), \\
& \mathbf{D}=\left(\begin{array}{cc}
i & -1 \\
k & 0 \\
0 & j \\
-1 & 0
\end{array}\right) \text {. }
\end{aligned}
$$

Since the following matrices are Hermitian:

$$
\begin{aligned}
& \mathbf{V}=\mathbf{A W}_{1}=\left(\begin{array}{ccc}
-2 & i & 0 \\
-i & -1 & 0 \\
0 & 0 & 0
\end{array}\right), \\
& \mathbf{U}=\mathbf{W}_{2} \mathbf{B}=\left(\begin{array}{ccc}
0 & -i & -i \\
i & -1 & 0 \\
i & 0 & -1
\end{array}\right),
\end{aligned}
$$

then we can find the $W$-weighted Drazin inverse solution of (78) by its determinantal representation (38).

We have

$$
\begin{aligned}
& k_{1}=\max \left\{\operatorname{Ind}\left(\mathbf{A W}_{1}\right), \operatorname{Ind}\left(\mathbf{W}_{1} \mathbf{A}\right)\right\}=1, \\
& k_{2}=\max \left\{\operatorname{Ind}\left(\mathbf{B W}_{2}\right), \operatorname{Ind}\left(\mathbf{W}_{2} \mathbf{B}\right)\right\}=1,
\end{aligned}
$$

and $s_{1}=\operatorname{rank}\left(\mathbf{A W}_{1}\right)=2$ and $s_{2}=\operatorname{rank}\left(\mathbf{W}_{2} \mathbf{B}\right)=2$. Since

$$
\left(\mathrm{AW}_{1}\right)^{3}=\left(\begin{array}{ccc}
-13 & 8 i & 0 \\
-8 i & -5 & 0 \\
0 & 0 & 0
\end{array}\right)
$$

$$
\left(\mathbf{W}_{2} \mathbf{B}\right)^{3}=\left(\begin{array}{ccc}
0 & -3 i & -3 i \\
3 i & -3 & 0 \\
3 i & 0 & 3
\end{array}\right)
$$

then

$$
\begin{aligned}
& \left.\sum_{\beta \in J_{2,3}} \mid\left((\mathbf{A W})_{1}\right)^{3}\right)_{\beta}^{\beta} \mid=1, \\
& \sum_{\alpha \in I_{2,3}}\left|\left(\left(\mathbf{W}_{2} \mathbf{B}\right)^{3}\right)_{\alpha}^{\alpha}\right|=-27 .
\end{aligned}
$$

We have

$$
\begin{aligned}
\overline{\mathbf{D}} & =\mathbf{A W}_{1} \mathbf{A D B W}_{2} \mathbf{B} \\
& =\left(\begin{array}{ccc}
2 i+j & -7+k & -5+2 k \\
-1+k & -5 i-j & -4 i-2 j \\
0 & 0 & 0
\end{array}\right) .
\end{aligned}
$$

By (40), we can get

$$
\begin{aligned}
& \mathbf{d}_{\cdot 1}^{\mathbf{B}}=\left(\begin{array}{c}
36 i-9 j \\
-27-9 k \\
0
\end{array}\right), \\
& \mathbf{d}_{\cdot 2}^{\mathbf{B}}=\left(\begin{array}{c}
-27 \\
-18 i \\
0
\end{array}\right), \\
& \mathbf{d}_{\cdot 3}^{\mathbf{B}}=\left(\begin{array}{c}
9-9 k \\
9 i+3 j \\
0
\end{array}\right) .
\end{aligned}
$$

Since

$$
\left(\mathrm{AW}_{1}\right)_{\cdot 1}^{3}\left(\mathbf{d}_{\cdot 1}^{\mathbf{B}}\right)=\left(\begin{array}{ccc}
36 i-9 j & 8 i & 0 \\
-27-9 k & -5 & 0 \\
0 & 0 & 4
\end{array}\right)
$$

then finally we obtain

$$
\begin{aligned}
x_{11} & =\frac{\sum_{\beta \in J_{2,3}\{1\}} \operatorname{cdet}_{1}\left(\left(\mathbf{A W}_{1}\right)_{\cdot 1}^{3}\left(\mathbf{d}_{\cdot 1}^{\mathbf{B}}\right)\right)_{\beta}^{\beta}}{\sum_{\beta \in J_{2,3}}\left|\left(\left(\mathbf{A W}_{1}\right)^{3}\right)_{\beta}^{\beta}\right| \sum_{\alpha \in I_{2,3}}\left|\left(\left(\mathbf{W}_{2} \mathbf{B}\right)^{3}\right)_{\alpha}^{\alpha}\right|} \\
& =\frac{36 i-27 j}{-27}=\frac{-4 i+3 j}{3} .
\end{aligned}
$$


Similarly,

$$
\begin{aligned}
& x_{12}=\frac{\operatorname{cdet}_{1}\left(\begin{array}{cc}
-27 & 8 i \\
-18 i & -5
\end{array}\right)}{-27}=\frac{1}{3} \text {, } \\
& x_{13}=\frac{\operatorname{cdet}_{1}\left(\begin{array}{cc}
9-9 k & 8 i \\
9 i-3 j & -5
\end{array}\right)}{-27}=\frac{-9-7 k}{9}, \\
& x_{21}=\frac{\operatorname{cdet}_{2}\left(\begin{array}{cc}
-13 & 36 i-9 j \\
-8 i & -27-9 k
\end{array}\right)}{-27}=\frac{-7-5 k}{3}, \\
& x_{22}=\frac{\operatorname{cdet}_{2}\left(\begin{array}{cc}
-13 & -27 \\
-8 i & -18 i
\end{array}\right)}{-27}=\frac{-2 i}{3} \text {, } \\
& x_{23}=\frac{\operatorname{cdet}_{2}\left(\begin{array}{cc}
-13 & -9-9 k \\
-8 i & 9 i+3 j
\end{array}\right)}{-27}=\frac{15 i-11 j}{9} \text {, } \\
& x_{31}=x_{32}=x_{33}=0 \text {. }
\end{aligned}
$$

So, the $W$-weighted Drazin inverse solution of (78) is

$$
\mathbf{X}=\frac{1}{9}\left(\begin{array}{ccc}
-12 i+9 j & 3 & -9-7 k \\
-21-15 k & -6 i & 15 i-11 j \\
0 & 0 & 0
\end{array}\right)
$$

Note that we used Maple with the package CLIFFORD in the calculations.

\section{Competing Interests}

The author declares that there are no competing interests.

\section{References}

[1] S. L. Adler, Quaternionic Quantum Mechanics and Quantum Fields, Oxford University Press, New York, NY, USA, 1995.

[2] J. D. Gibbon, "A quaternionic structure in the threedimensional Euler and ideal magneto-hydrodynamics equations," Physica D, vol. 166, no. 1-2, pp. 17-28, 2002.

[3] J. D. Gibbon, D. D. Holm, R. M. Kerr, and I. Roulstone, "Quaternions and particle dynamics in the Euler fluid equations," Nonlinearity, vol. 19, no. 8, pp. 1969-1983, 2006.

[4] A. J. Hanson and H. Ma, "Quaternion frame approach to streamline visualization," IEEE Transactions on Visualization and Computer Graphics, vol. 1, no. 2, pp. 164-174, 1995.

[5] B. L. Stevens, F. L. Lewis, and E. N. Johnson, Aircraft Control and Simulation: Dynamics, Controls Design, and Autonomous Systems, John Wiley \& Sons, 3rd edition, 2015.

[6] A. Perez and J. M. McCarthy, "Dual quaternion synthesis of constrained robotic systems," Journal of Mechanical Design, vol. 126, no. 3, pp. 425-435, 2004.

[7] T. Isokawa, T. Kusakabe, N. Matsui, and F. Peper, "Quaternion neural network and its application," in Knowledge-Based Intelligent Information and Engineering Systems: 7th International Conference, KES 2003, Oxford, UK, September 2003. Proceedings, Part II, vol. 2774 of Lecture Notes in Computer Science, pp. 318-324, Springer, Berlin, Germany, 2003.

[8] J. Prošková, "Description of protein secondary structure using dual quaternions," Journal of Molecular Structure, vol. 1076, pp. 89-93, 2014.
[9] S. F. Yuan, Q. W. Wang, and X. F. Duan, "On solutions of the quaternion matrix equation $A X=B$ and their applications in color image restoration," Applied Mathematics and Computation, vol. 221, pp. 10-20, 2013.

[10] Q. Wang, S. Yu, and W. Xie, "Extreme ranks of real matrices in solution of the quaternion matrix equation $A X B=C$ with applications," Algebra Colloquium, vol. 17, no. 2, pp. 345-360, 2010.

[11] S. Yuan, A. Liao, and Y. Lei, "Least squares Hermitian solution of the matrix equation $(A X B ; C X D)=(E ; F)$ with the least norm over the skew field of quaternions," Mathematical and Computer Modelling, vol. 48, pp. 91-100, 2008.

[12] L. G. Feng and W. Cheng, "The solution set to the quaternion matrix equation $A X-X B=0$," Algebra Colloquium, vol. 19, no. 1, pp. 175-180, 2012.

[13] T. S. Jiang and M. S. Wei, "On a solution of the quaternion matrix equation $X-A \widetilde{X} B=C$," Acta Mathematica Sinica, vol. 21, no. 3, pp. 483-490, 2005.

[14] S. Caiqin, C. Guoliang, and W. Xiaodong, "On solutions of quaternion matrix equations $X F \# A X=B Y$ and $X F \# A \widetilde{X}=B Y$," Acta Mathematica Scientia, vol. 32, no. 5, pp. 1967-1982, 2012.

[15] S.-F. Yuan and Q.-W. Wang, "Two special kinds of least squares solutions for the quaternion matrix equation $A X B+C X D=E$," Electronic Journal of Linear Algebra, vol. 23, pp. 257-274, 2012.

[16] F. Zhang, M. Wei, Y. Lia, and J. Zhao, "Special least squares solutions of the quaternion matrix equation $A X=B$ with applications," Applied Mathematics and Computation, vol. 270, pp. 425-433, 2015.

[17] R. E. Cline and T. N. E. Greville, "A Drazin inverse for rectangular matrices," Linear Algebra and Its Applications, vol. 29, pp. 53-62, 1980.

[18] S. L. Campbell, C. D. Meyer Jr., and N. J. Rose, “Applications of the Drazin inverse to linear systems of differential equations with singular constant coefficients," SIAM Journal on Applied Mathematics, vol. 31, no. 3, pp. 411-425, 1976.

[19] S. L. Campbell, "Comments on 2-D descriptor systems," Automatica, vol. 27, no. 1, pp. 189-192, 1991.

[20] S. L. Campbell and C. D. Meyer, Generalized Inverse of Linear Transformations. Corrected Reprint of the 1979 Original, Dover, New York, NY, USA, 1991.

[21] D. J. Spitzner and T. R. Boucher, "Asymptotic variance of functionals of discrete-time Markov chains via the Drazin inverse," Electronic Communications in Probability, vol. 12, pp. 120-133, 2007.

[22] T. Kaczorek, "Application of the Drazin inverse to the analysis of descriptor fractional discrete-time linear systems with regular pencils," International Journal of Applied Mathematics and Computer Science, vol. 23, no. 1, pp. 29-33, 2013.

[23] Z. Al-Zhour, A. Kılıçman, and M. H. Abu Hassa, "New representations for weighted Drazin inverse of matrices," International Journal of Mathematical Analysis, vol. 1, no. 15, pp. 697708, 2007.

[24] M. Nikuie and M. Z. Ahmad, "New results on the W-weighted drazin inverse," in Proceedings of the 3rd International Conference on Mathematical Sciences, vol. 1602 of AIP Conference Proceedings, p. 157, Kuala Lumpur, Malaysia, June 2014.

[25] M. Nikuie, "Singular fuzzy linear systems," Applied Mathematics and Computational Intelligence, vol. 2, no. 2, pp. 157-168, 2013.

[26] G. Wang and J. Sun, "A Cramer rule for solution of the general restricted matrix equation," Applied Mathematics and Computation, vol. 154, no. 2, pp. 415-422, 2004. 
[27] Y. Wei, "A characterization for the W-weighted Drazin inverse and a Cramer rule for the W-weighted Drazin inverse solution," Applied Mathematics and Computation, vol. 125, no. 2-3, pp. 303-310, 2002.

[28] G. J. Song, "Characterization of the W-weighted Drazin inverse over the quaternion skew field with applications," Electronic Journal of Linear Algebra, vol. 26, pp. 1-14, 2013.

[29] I. Kyrchei, "Determinantal representations of the W-weighted Drazin inverse over the quaternion skew field," Applied Mathematics and Computation, vol. 264, Article ID 21118, pp. 453-465, 2015.

[30] I. Kyrchei, "Cramer's rule for quaternion systems of linear equations," Journal of Mathematical Sciences, vol. 155, no. 6, pp. 839-858, 2008.

[31] I. Kyrchei, "The theory of the column and row determinants in a quaternion linear algebra," in Advances in Mathematics Research, A. R. Baswell, Ed., vol. 15, pp. 301-359, Nova Science, New York, NY, USA, 2012.

[32] I. I. Kyrchei, "Cramer's rule for some quaternion matrix equations," Applied Mathematics and Computation, vol. 217, no. 5, pp. 2024-2030, 2010.

[33] I. I. Kyrchei, "Determinantal representations of the MoorePenrose inverse over the quaternion skew field and corresponding Cramer's rules," Linear and Multilinear Algebra, vol. 59, no. 4, pp. 413-431, 2011.

[34] I. Kyrchei, "Determinantal representation of the MoorePenrose inverse matrix over the quaternion skew field," Journal of Mathematical Sciences, vol. 180, no. 1, pp. 23-33, 2012.

[35] I. Kyrchei, "Explicit representation formulas for the minimum norm least squares solutions of some quaternion matrix equations," Linear Algebra and Its Applications, vol. 438, no. 1, pp. 136-152, 2013.

[36] I. Kyrchei, "Determinantal representations of the Drazin inverse over the quaternion skew field with applications to some matrix equations," Applied Mathematics and Computation, vol. 238, pp. 193-207, 2014.

[37] G.-J. Song, "Determinantal representation of the generalized inverses over the quaternion skew field with applications," Applied Mathematics and Computation, vol. 219, no. 2, pp. 656667, 2012.

[38] G.-J. Song, "Bott-Duffin inverse over the quaternion skew field with applications," Journal of Applied Mathematics and Computing, vol. 41, no. 1-2, pp. 377-392, 2013.

[39] G.-J. Song, Q.-W. Wang, and H.-X. Chang, "Cramer rule for the unique solution of restricted matrix equations over the quaternion skew field," Computers \& Mathematics with Applications, vol. 61, no. 6, pp. 1576-1589, 2011.

[40] C. Song, X. Wang, and X. Zhang, "On solutions of the generalized Stein quaternion matrix equation," Journal of Applied Mathematics and Computing, vol. 43, no. 1-2, pp. 115-131, 2013.

[41] V. Rakočević and Y. Wei, "A weighted Drazin inverse and applications," Linear Algebra and Its Applications, vol. 350, pp. 25-39, 2002.

[42] Y. Wei, "Integral representation of the W-weighted Drazin inverse," Applied Mathematics and Computation, vol. 144, pp. 310, 2003. 


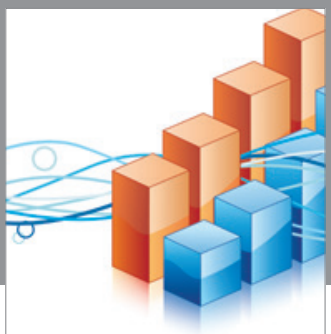

Advances in

Operations Research

vatem alat4

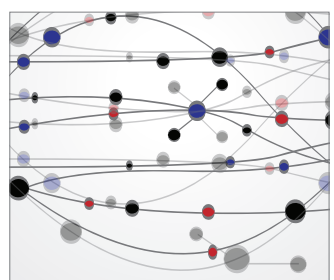

\section{The Scientific} World Journal
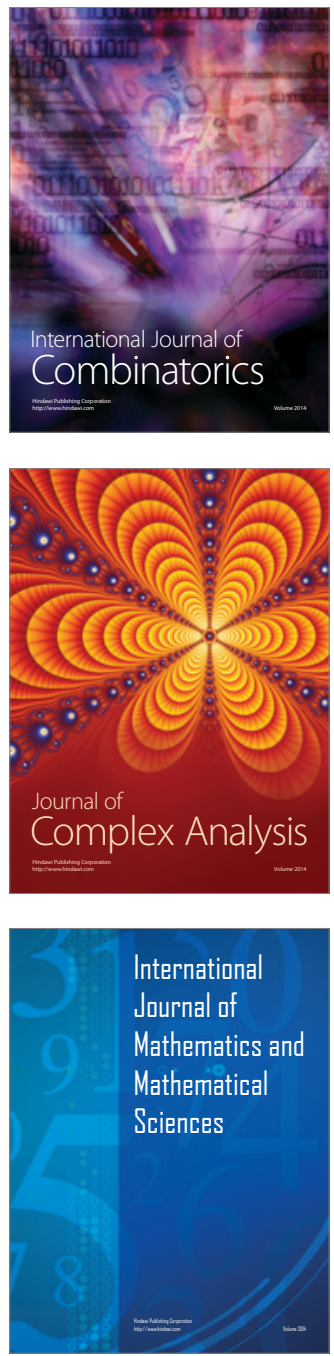
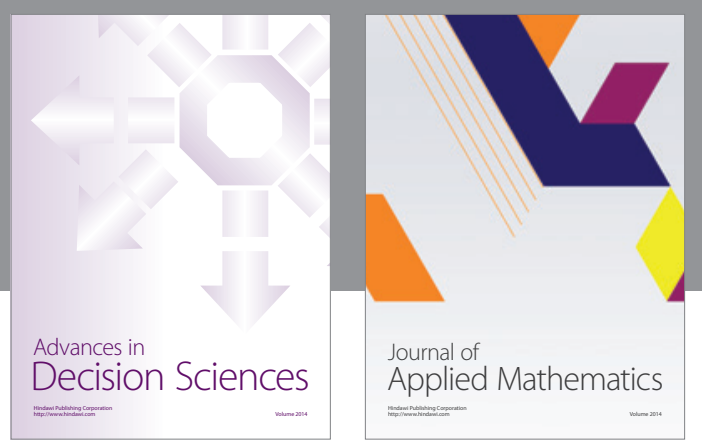

Algebra

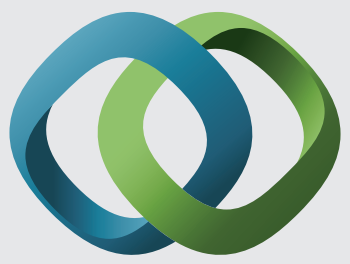

\section{Hindawi}

Submit your manuscripts at

http://www.hindawi.com
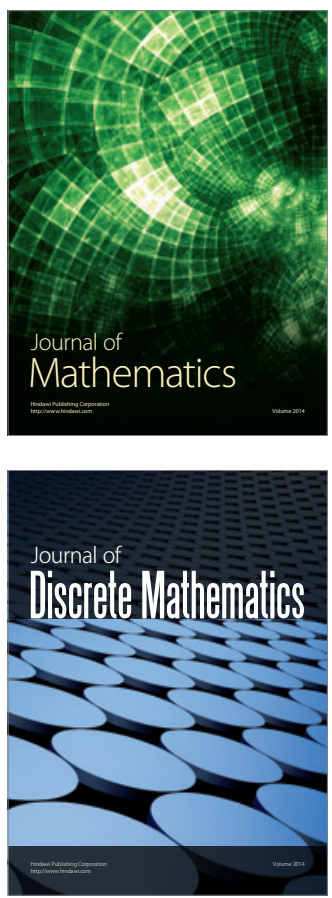

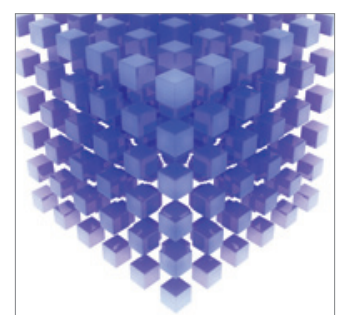

Mathematical Problems in Engineering
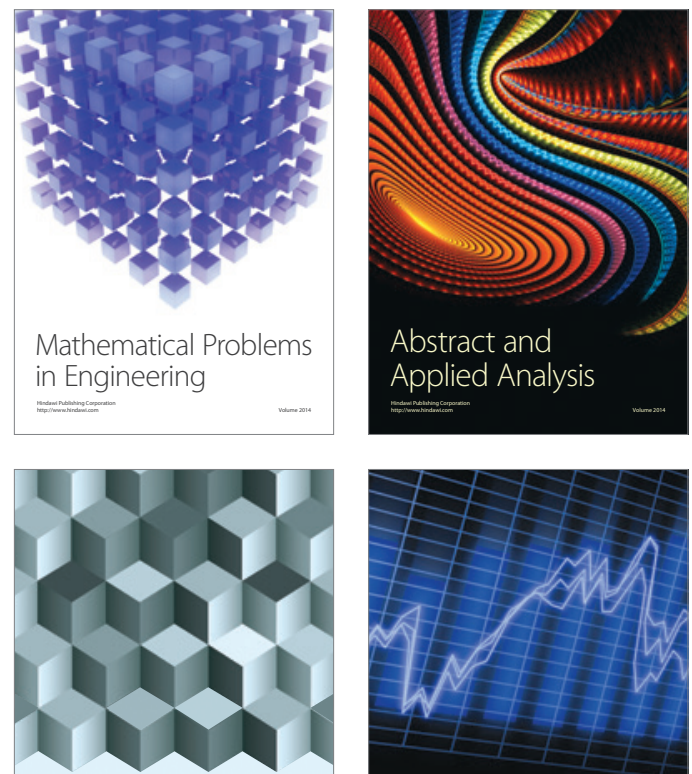

Journal of

Function Spaces

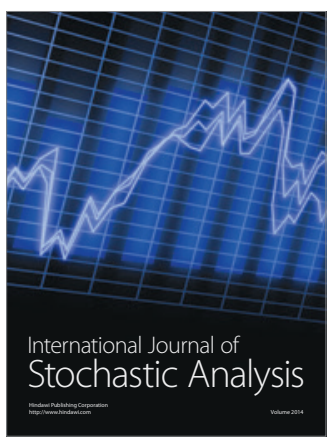

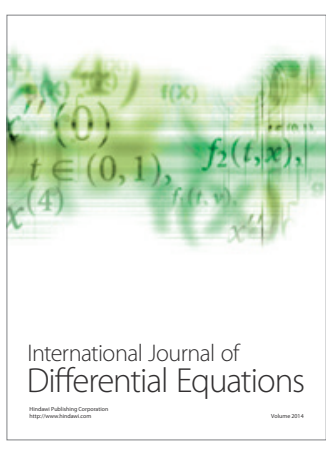
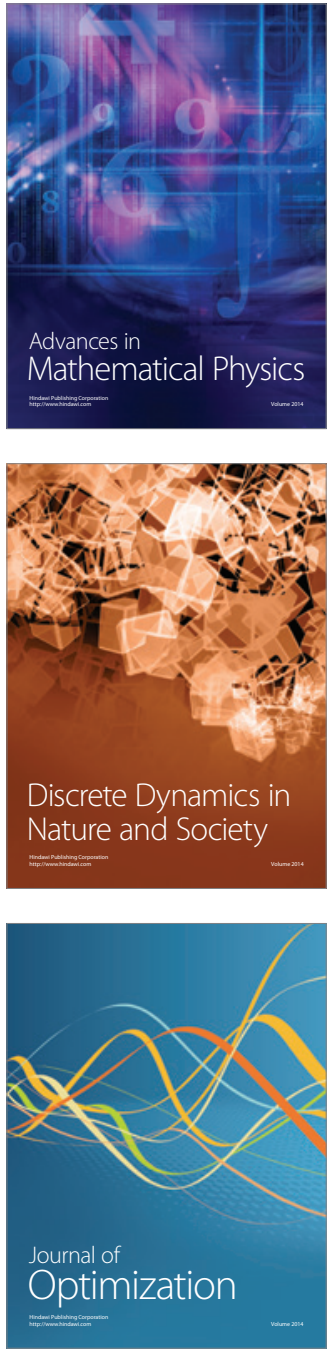